\title{
Radicalisation djihadiste et discours victimaire sur les réseaux sociaux : de la victime au bourreau
}

Jihadist Radicalization and Victim-Discourse on social Media: From Victim to Executioner

\section{Laurène Renaut}

\section{(2) OpenEdition}

\section{Journals}

Édition électronique

URL : http://journals.openedition.org/aad/3870

DOI : 10.4000/aad.3870

ISSN : 1565-8961

Éditeur

Université de Tel-Aviv

Référence électronique

Laurène Renaut, «Radicalisation djihadiste et discours victimaire sur les réseaux sociaux : de la victime au bourreau », Argumentation et Analyse du Discours [En ligne], 23 | 2019, mis en ligne le 17 octobre 2019, consulté le 03 décembre 2019. URL : http://journals.openedition.org/aad/3870 ; DOI 10.4000/aad.3870

Ce document a été généré automatiquement le 3 décembre 2019.

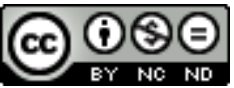

Argumentation \& analyse du discours est mis à disposition selon les termes de la licence Creative Commons Attribution - Pas d'Utilisation Commerciale - Pas de Modification 4.0 International. 


\title{
Radicalisation djihadiste et discours victimaire sur les réseaux sociaux : de la victime au bourreau
}

Jihadist Radicalization and Victim-Discourse on social Media: From Victim to

\author{
Executioner
}

Laurène Renaut

\section{Introduction}

Mars 2004 à Madrid, juillet 2005 à Londres, novembre 2015 à Paris... Les dix-huit dernières années ont été marquées par une succession d'attaques terroristes en Europe. Face à la violence et à la complexité de la radicalisation djihadiste, les études sur ce sujet se multiplient. A cet égard, si plusieurs financements ont favorisé l'émergence d'une réflexion interdisciplinaire, la recherche s'est focalisée jusqu'à présent sur l'étude du contexte psycho-sociologique dans lequel se développe ce phénomène (Khosrokhavar 2014, Benslama 2016) ainsi que sur les stratégies adoptées par Daesh pour recruter ses sympathisants (Rogan 2006). Or, comme montré par Ascone (2018), nombreux sont les éléments qui soulignent l'importance du langage dans la radicalisation, le discours étant le vecteur de toute idéologie (Althusser 1970, Pêcheux, 1975, Reboul, 1980). Autrement dit, pour comprendre les ressorts de l'idéologie djihadiste, c'est à dire les aspirations et les mécanismes de passage à l'acte sur lesquels elle repose, il semble essentiel d'examiner comment cette idéologie est mise en discours par ses sympathisants. C'est précisément dans ce contexte, où le rôle du langage semble déterminant, que se développe cette étude. Elle se propose plus exactement d'interroger, d'un point de vue discursif, la construction du dispositif victimaire djihadiste et de montrer comment celui-ci est mis au service d'une justification de l'action violente. En effet, si nous avons choisi de nous pencher sur la posture de victime dans le cadre du djihadisme, c'est bien parce que la victimisation 
occupe une place prépondérante tant dans la propagande de Daesh qu'à toutes les étapes du processus de radicalisation ${ }^{1}$.

Compte tenu de la diversité des corpus mobilisables pour une telle problématique, nous avons choisi de centrer notre propos sur le discours numérique de profils caractérisés comme radicalisés. La première partie de l'article explicite la démarche adoptée, l'adossement théorique ainsi que méthodologique, et propose une caractérisation thématique du corpus à partir de la notion de grief. Dans une seconde partie, nous analysons les ressorts sémio-discursifs sur lesquels repose la construction d'une identité victimaire, autrement dit d'un ethos de victime, dans la djihadosphère. Enfin, nous montrons dans quelle mesure le discours de victimisation djihadiste est mis au service d'une justification de la violence par la construction d'un ethos de justicier.

\section{Construction discursive de la victime : pertinence et méthode de saisie de la victimisation}

3 L'approche sémio-discursive que nous proposons s'appuie sur le relevé de marques linguistiques et sémiotiques, mais se heurte à un paradoxe apparent. En effet, qui dit victimisation, dit victime, et plus précisément sentiment d'être victime. Or le terme " victime » n'apparaît pas dans notre corpus ${ }^{2}$, et la question qui se pose alors est de comprendre les processus de sémiotisation du statut de victime sans passer par le recours au terme explicite.

\section{1. «L'idéal islamique blessé » comme voie de victimisation}

4 En effet, l'état victimaire dont il est question dans cette recherche n'est pas le produit d'un constat ou d'un fait objectivement démontrable. Ici, « être une victime devient la modalité expressive d'un traumatisme subjectivement ressenti» (Messu 2018: 94); autrement dit, le vocable « victime » relève avant tout du sentiment subjectif, d'où la désignation dans ce cas particulier de "victime subjective " ou "victime identitaire ", entendue au sens d'une "personne éprouvant un sentiment victimaire lié à un ordre social injuste et se réclamant d'une identité de victime " (ibid.). Or, dans les cas de victimisation auxquels nous nous intéressons, le terme "victime» est absent des productions analysées. La saisie de la victimisation dans le corpus doit donc se faire non pas à partir du repérage d'un mot-pivot ("victime»), ni à partir de la description des profils comme victimes, mais à partir de la caractérisation d'une interprétation de victime sans l'usage du terme lui-même.

Si l'étude des causes ou facteurs favorisant ce ressenti ne sera pas l'objet de notre analyse, quelques hypothèses tant d'un point de vue historique que psychologique ont été récemment soulevées sur le sujet. En effet, comme le note Keane (2015), le terrorisme djihadiste a pour caractéristique de présenter la communauté musulmane comme victime de l'Occident. Né de facteurs politiques, sociaux, économiques et religieux, il se développe suite à trois évènements consécutifs qui vont dans ce sens : l'invasion soviétique de l'Afghanistan à la fin des années 70, l'exécution de Saddam Hussein en 2006, et l'émergence en 2011 d'une guerre civile en Syrie ${ }^{3}$. Dans cette perspective, ses recrues sont incitées à prendre les armes pour reconquérir leur liberté. 
6 Ces éléments rejoignent ainsi une forme d'idéologie de l'«idéal islamique blessé » mise en lumière par Benslama (2016) et sur laquelle reposerait le discours djihadiste. Cette blessure serait liée à la rencontre entre le monde de l'islam et la modernité occidentale, laquelle sépare le politique du religieux, éloignant l'individu de Dieu et de la communauté. Fethi Benslama pointe le caractère scientifique et militaire des expéditions menées dans les pays de civilisation musulmane à la fin du $18^{\mathrm{e}}$ siècle et placées sous le signe d'une domination suscitant aussi bien fascination qu'humiliation. La fin du califat ottoman en 1924 achève ensuite cette séparation du religieux et du politique avec l'instauration du premier État laïque en Turquie, vécue comme une catastrophe, et suivie de l'émergence du mouvement des Frères musulmans en 1928. Le discours islamiste va ainsi s'appuyer sur la paupérisation des pays de confession musulmane et sur une dénonciation des promesses de progrès liées à la modernité occidentale. Restent dès lors la solution religieuse et l'adoption de la figure du "surmusulman", définie comme "la manière d'être qui surgit à la croisée d'un parcours personnel où la souffrance et/ ou le manque de reconnaissance prédominent, et d'un discours de l'idéal islamique blessé » (Lahrache 2016:12). Il s'agirait dès lors pour le « surmusulman » de " prendre sur soi la réparation de l'affront fait à l'islam [...] et qui se perpétuerait de génération en génération » (ibid.).

7 C'est dans ce contexte que nous nous proposons d'étudier les modalités d'expression de cet "idéal islamique blessé ", autrement dit les tentatives en discours de s'ajuster aux attendus de la figure générique de victime, et cela à travers les données publiques de vingt profils radicalisés sur Facebook (dix hommes et dix femmes); donc via un corpus numérique anonymisé pour des raisons de sécurité comme de confidentialité. Précisons qu'une grille de critères a été préétablie dans le cadre de cette recherche afin de définir les éléments caractéristiques attestant d'une forme de radicalisation sur ce réseau social :

\begin{tabular}{|c|c|c|c|}
\hline & $\begin{array}{l}\text { Contenus investis (catégories/ } \\
\text { thèmes) }\end{array}$ & Symbolique & $\begin{array}{l}\text { Mots-clés } \\
\text { récurrents }\end{array}$ \\
\hline $\begin{array}{l}\text { Critères } \\
\text { déterminants }\end{array}$ & $\begin{array}{l}\text { Apologie explicite ou implicite du } \\
\text { terrorisme djihadiste } \\
\text { Hommage aux martyrs } \\
\text { Partage de supports de propagande } \\
\text { diffusés par Al-Hayat Media Center } \\
\text { Soutien des actions militaires de } \\
\text { Daesh }\end{array}$ & $\begin{array}{l}\text { Drapeau d'une organisation } \\
\text { terroriste } \\
\text { Photos de combattants } \\
\text { Extrait d'anasheed }{ }^{5} \text { djihadiste } \\
\text { suivi d'une note de musique }\end{array}$ & $\begin{array}{l}\text { Slogan de l'EI : } \\
\text { « Keep calm } \\
\text { and say } \\
\text { Baraqiya » }\end{array}$ \\
\hline
\end{tabular}

8 Cette grille a été établie lors d'un travail de parcours et de recension susceptibles d'être caractérisés comme radicalisés, en faisant attention à cerner précisément cette catégorie en comparaison à d'autres catégories parfois proches sur certains aspects (comme certains comptes salafistes). Ces profils ont pour dénominateur commun de présenter des discours d'anonymes en voie de radicalisation, c'est à dire des profils se distinguant par leur adhésion explicite ou implicite à l'idéologie djihadiste, et cela à des stades différents d'engagement ( $\mathrm{du}$ sympathisant de Daesh, à des veuves de combattants sur zones, jusqu'à un terroriste présumé incarcéré à Fresnes). Précisons 
qu'aucun de ces profils ne constitue un compte de propagande officielle pour Daesh même si certains d'entre eux en deviennent des relais indirects.

\subsection{Travailler le corpus Facebook comme lieu de victimisation : méthodes et enjeux}

Le terrain de notre analyse est donc l'espace numérique constitué par les comptes de ces vingt profils, et cet espace est dynamique : il génère de l'interaction, intègre des liens, reprend des éléments circulants. Le corpus est donc considéré comme un «terrain» (Longhi 2018), qui a été préparé en amont par l'observation et l'établissement de questions de recherche préalables. Ce terrain peut ensuite être investi, et questionné avec les outils offerts par une analyse sémiolinguistique. Si un tel corpus est considéré comme un terrain, il n'est donc pas exhaustif, ni exclusif d'autres corpus. En travaillant au cœur de ces espaces hypertextualisés, lesquels induisent une ouverture du corpus vers des sites, des vidéos ou d'autres comptes, nous nous intégrons donc dans une méthodologie de la circulation du sens et de la prise en compte de l'interdiscours (Garric et Longhi 2012) ${ }^{6}$.

10 Concernant plus spécifiquement le recours aux réseaux sociaux (qui n'est pas l'unique manière d'aborder la thématique, mais une voie d'accès spécifique, et pertinente, à certains fonctionnements), il se justifie dans la mesure où être une victime passe par une image de soi qui est donnée à voir aux autres comme à soi-même : c'est « un fairevaloir qui doit s'énoncer face à tous les autres» (Messu 2018: 89). Cette hypothèse est renforcée par les propos de Paveau (2012) à propos de Facebook questionné comme un espace monologique permettant «une forme de communication qui met le sujet du discours au centre de sa propre parole et de celle des autres, et qui utilise l'internet comme une source permanente et toujours disponible de confirmation narcissique " (https://penseedudiscours.hypotheses.org/235). Ce terrain est donc potentiellement riche pour notre problématique, puisqu'il propose un espace ouvert mais propice à l'expression des scripteurs centrés sur eux-mêmes, avec des effets de circulations, d'échos, d'emprunts à des discours et des ressources sémiotiques riches.

\subsection{Caractérisation thématique des griefs}

11 Le dispositif victimaire djihadiste relève d'abord d'un discours de légitimation des préjudices subis qui font ici l'objet de doléances sur les réseaux sociaux. En effet, «il revient à la victime subjective de transcender l'expression affective de son sentiment d'être une victime pour fournir les bonnes raisons, les raisons partagées, celles qui vont emporter la conviction, afin que la reconnaissance statutaire se fasse " (Messu 2018: 97). A cet égard, si la liste des griefs à l'égard des « mécréants $»^{7}$ est longue, les « bonnes raisons » en tant que motifs de reproche s'articulent principalement autour des trois valeurs républicaines (Liberté, Egalité, Fraternité) mais comprises ici dans leur négation; les profils radicalisés exprimant leur sentiment de ne pas être traités au regard de ces principes. 
1.3.1. De la société de surveillance à l'univers carcéral : une « liberté » à géométrie variable

12 Le premier sujet de plainte réside dans la dénonciation d'une privation de liberté qui se manifeste par une société de surveillance dont la communauté musulmane serait victime aux premières loges. En atteste la diffusion d'une image significative sur plusieurs comptes entre octobre-novembre 2017.

Image 1

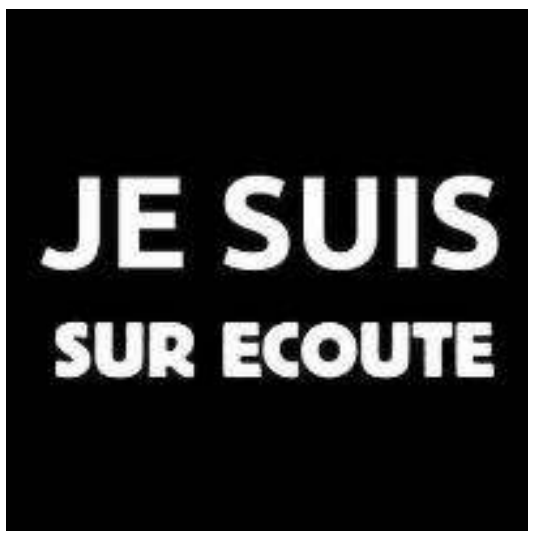

13 Cette image, relayée via le bouton de partage Facebook, compris au sens de «technosigne » par Paveau (2015), et recouvrant ici la fonction d'une photo de profil, réinvestit tous les codes sémio-discursifs (couleurs, taille de la police et caractère) du slogan «Je suis Charlie» diffusé après les attentats de janvier 2015. Cette forme de contre-attaque numérique constitue dès lors un signe visible de ralliement pour une " communauté communicante " (Aim et Escande Gauquié 2016) caractérisée par son adhésion à l'idéologie djihadiste. Au sentiment d'être surveillé se greffe celui d'être emprisonné injustement, la prison apparaissant d'ailleurs comme un leitmotiv sur ces véritables murs des lamentations :

(1) «La liberté d'expression est que pour ceux qui insultent l'islam, par contre les musulmans c'est le hebs ${ }^{8}$ qui les attend » (Post du 15/10/17). 
Image 2

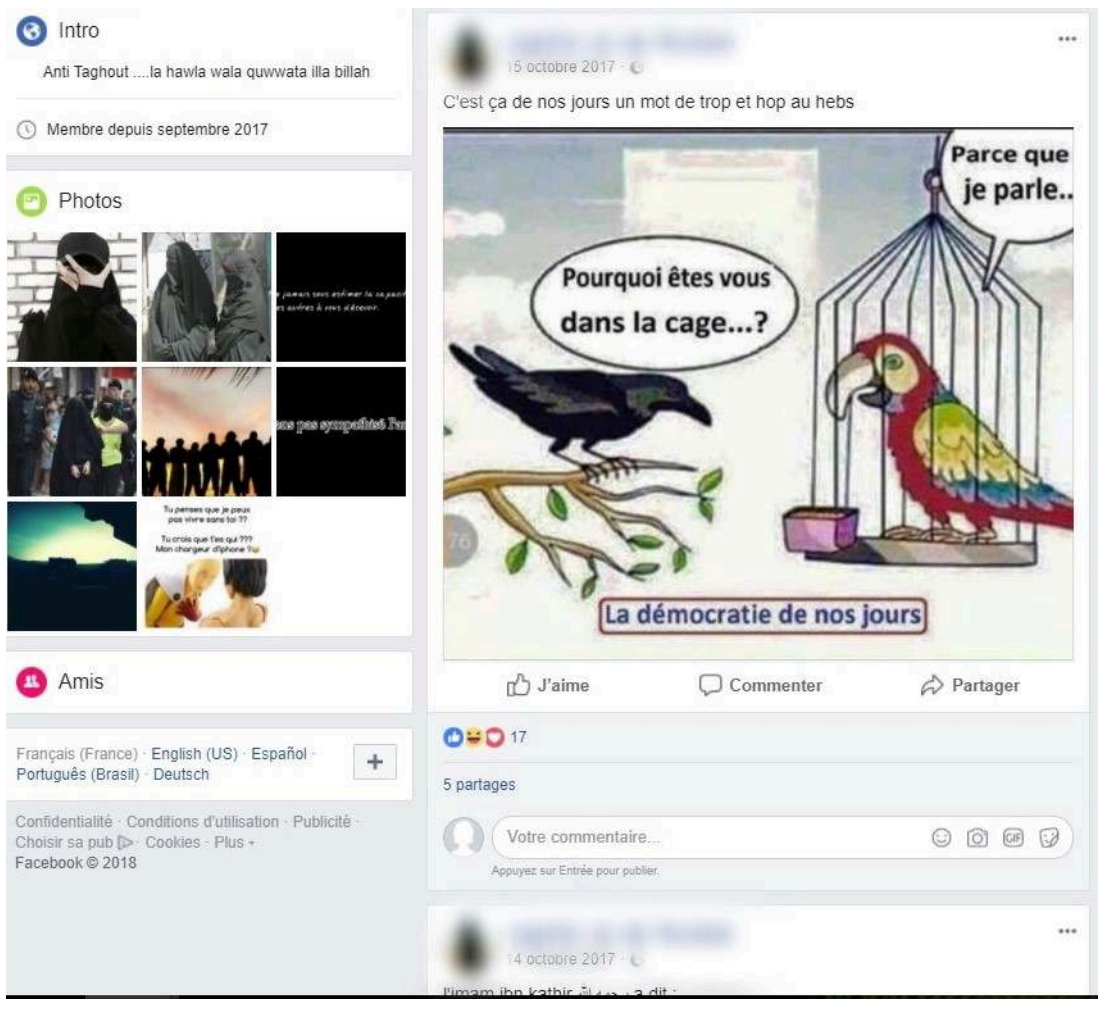

\subsubsection{La République comme terreau des discriminations : l'échec de l'Egalité}

14 Se présentant comme victimes de discriminations quotidiennes, les profils dénoncent les inégalités dont ils font l'objet dans les lieux mêmes de la République, notamment dans les écoles (exemple 2), devant la justice (image 3) mais aussi dans l'espace public avec le port de la burqa qui cristallise le ressentiment (image 4).

(2) « Je vais chercher ma nièce en larmes à la maternelle, la directrice me convoque dans son bureau. Et vous savez pourquoi ??? Parce que la petite a dit « BISMILLAH » avant de manger à la cantine ! :) » (Post du 15/12/17)

Commentaire 1: «La même directrice qui lui souhaitera JOYEUX NOEL avant les vacances et lui demandera à la rentrée quel cadeau elle a eu... »

Réponse $1:$ : Ils sont fous ces kuffars »!

Réponse $2:$ «Ils font exprès, moi une fois elle a dit à ma fille que dieu existe pas mais pour le père noël pas de problème, on leur retourne le cerveau.

Réponse 3 : «C'est des gros malades, wllahi, c'est écoeurant, qu'Allah les brise.» 
Image 3

ent $*$

alitult

23 mars, $14: 57 \cdot 6$

PTDRR qui a pu rentrer chez lui pour dormir, mangée, prendre sa douche tranquille pendant sa garde à vue ? hein bah pas moi en tout cas, justice a geometrie variable, encore du cinéma

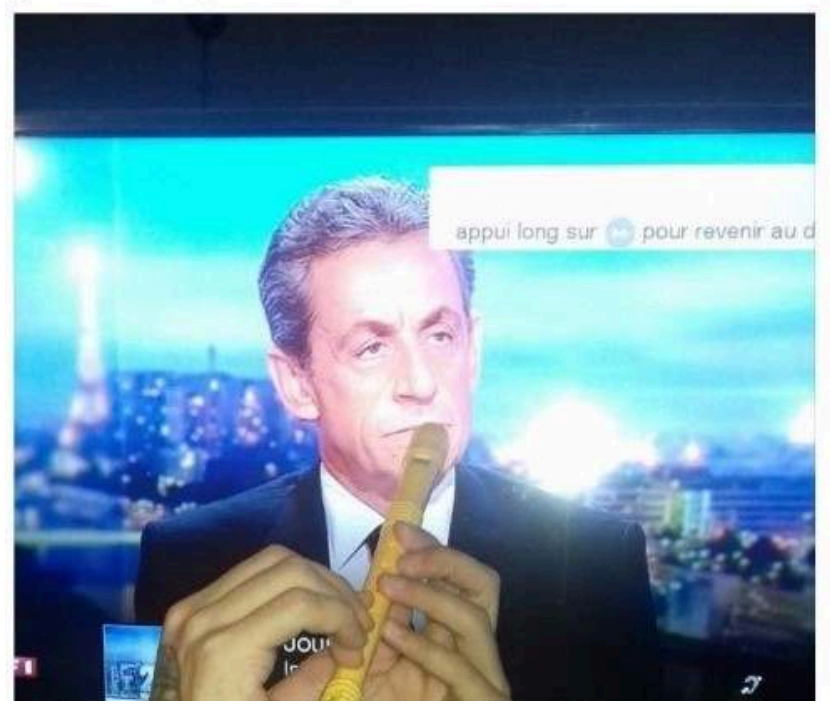

Image 4
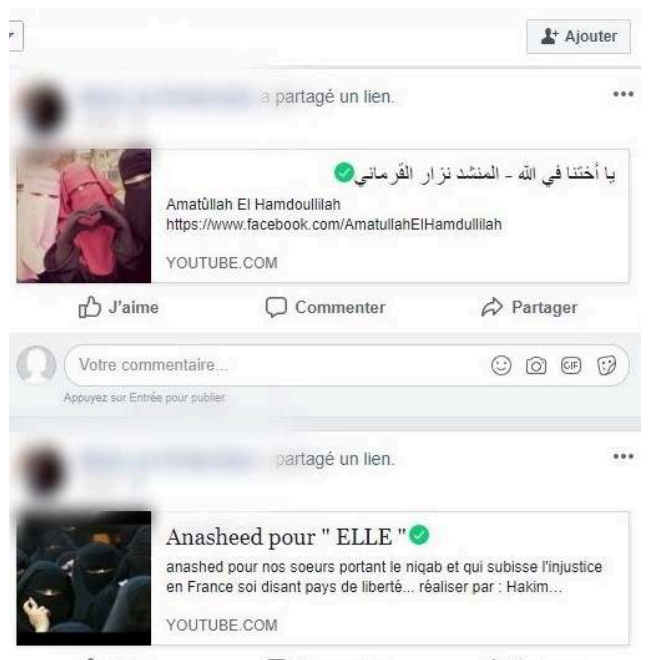

ه J'aime $\square$ Commenter $\Rightarrow$ Partager

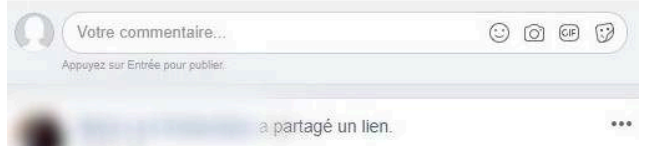

1 The Eternals 0

Пророк С.А.В Сказалл «Если у супругов взгляд друг к другу с
Любовью. то Аллах смотрит на них с милостью»

YOUTUBE.COM 


\subsubsection{Entre invisibilité médiatique et désinformation : le miroir inversé de la Fraternité}

Enfin, le sentiment d'un déficit de fraternité envers la communauté musulmane dans le monde se manifeste à travers une dénonciation du traitement médiatique occidental des souffrances qu'elle endure dans l'indifférence générale. Si cette critique concerne d'abord une absence de médiatisation avec comme principaux oubliés la Birmanie, la Palestine ou la Syrie (image 5), elle renvoie aussi à un phénomène de désinformation qui suscite l'indignation (image 6). La communauté musulmane souffrirait non seulement d'une invisibilité mais aussi d'une manipulation volontaire de l'information destinée à tromper l'opinion publique en la liguant contre les « musulmans ».

Image $5^{9}$

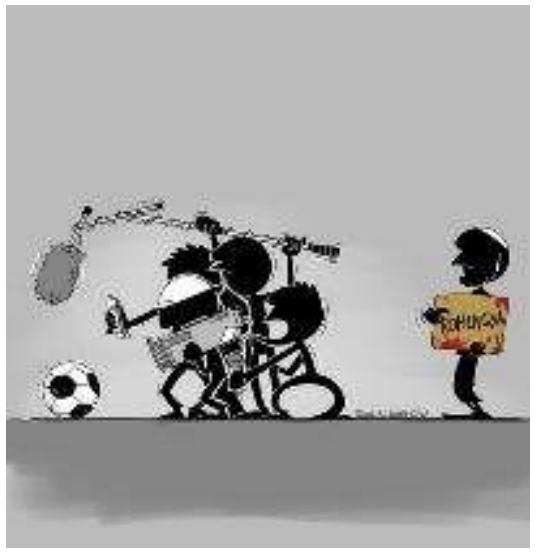

Image 6
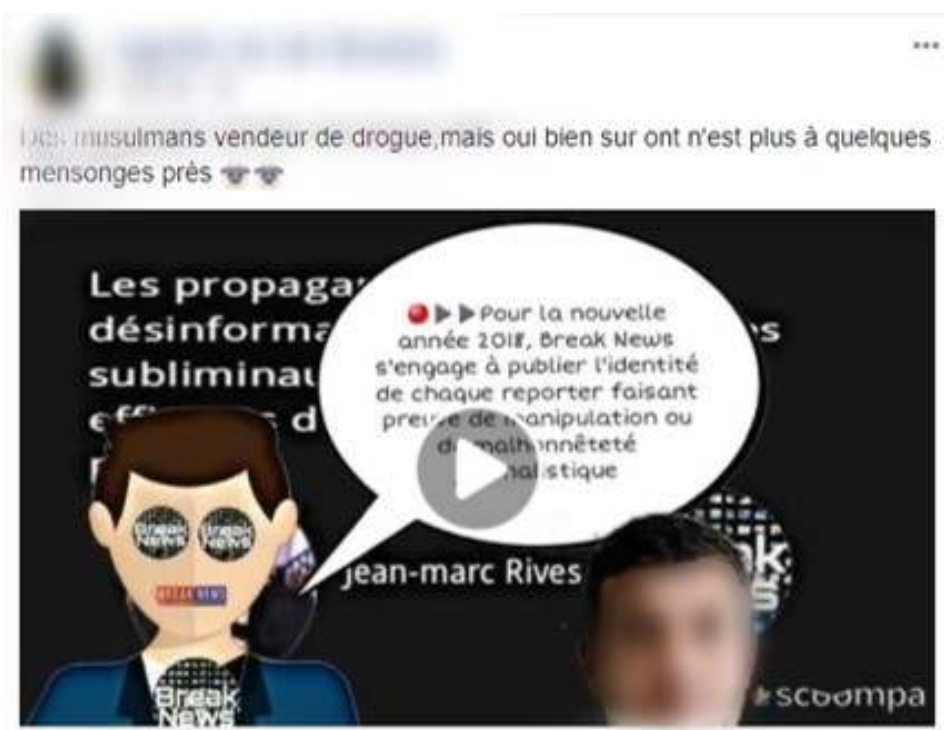

210 vuess

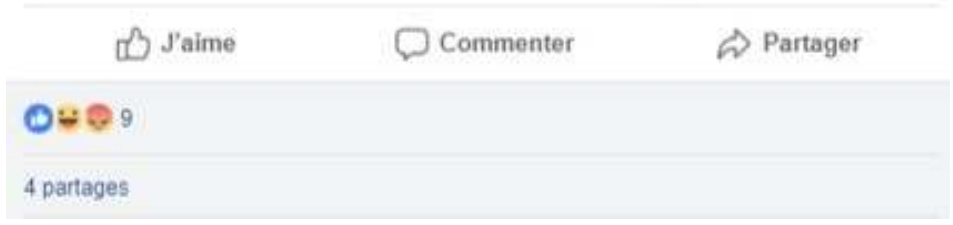


A partir de cette catégorisation thématique, distinguons trois formes de victimisation :

- une victimisation d'ordre verbal qui réfère à des attaques proférées par le biais des mots ne faisant pas spécifiquement ici l'objet de griefs

- une victimisation d'ordre physique qui renvoie à des comportements menaçant l'intégrité physique (Hawker et Boulton 2000) : image 5

- une victimisation d'ordre relationnel qui concerne les comportements favorisant un sentiment d'exclusion sociale (Crick et al. 1999), comme la propagation de rumeurs (Crick et Grotpeter, 1995) : images 2, 4, 6 et exemple 2.

À ces diverses modalités de victimisation s'ajoute une autre forme d'agression qui revient dans les témoignages des profils radicalisés et que l'on pourrait catégoriser comme une cyberintimidation, relevant d'une forme de censure sur les réseaux sociaux. En effet, il n'est pas rare de trouver des publications relatives à la fermeture répétée de leurs comptes, les obligeant à se camoufler en usant de pseudonymes variés ou en téléchargeant des applications sensés proposer des alternatives à Facebook et à ses instances de contrôle (image 7).

Image 7

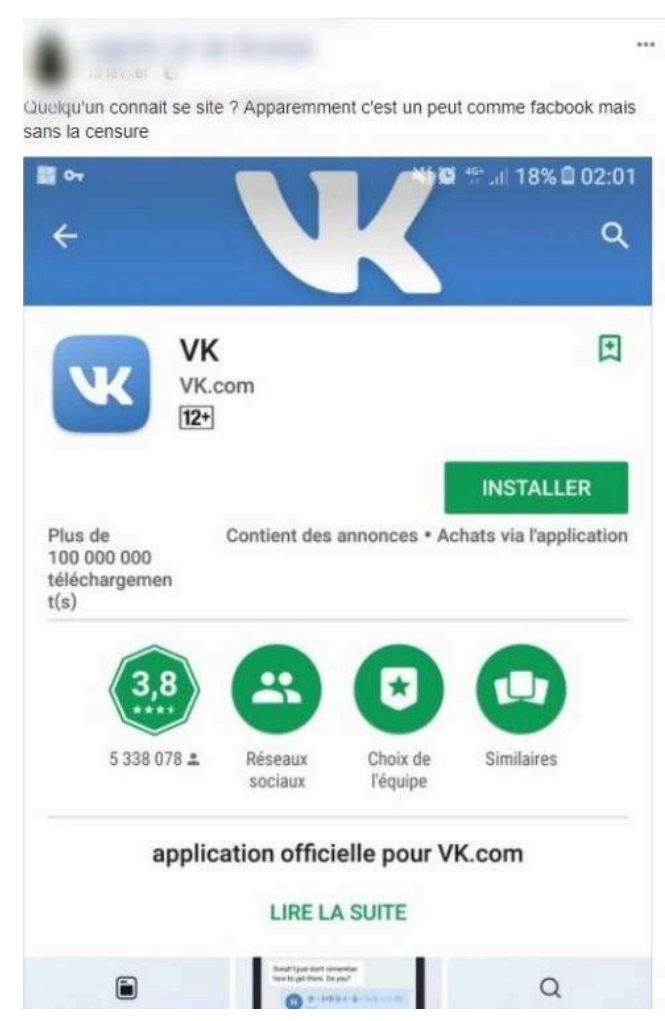

\section{S'ériger en victime : la construction d'une identité victimaire}

La mise en œuvre discursive de soi repose ici sur la construction d'un ethos implicite de victime; implicite car si «l'ethos se montre dans l'acte d'énonciation, il ne se dit pas dans l'énoncé. Il reste par nature au second plan de l'énonciation : il doit être perçu, mais ne pas faire l'objet du discours » (Maingueneau, 2013 : 10). Dès lors, on peut parler d'un ethos discursif «montré » et non pas « dit » puisqu'à aucun moment l'énonciateur 
n'évoque sa propre énonciation: en effet on ne relève aucune occurrence du mot "victime » sur les profils qui font l'objet de notre recherche. Pour autant, ces derniers s'appuient tous sur une rhétorique de la victimisation dont la mécanique constitue un levier identitaire mais aussi un moyen d'affirmation comme de revendication.

Dans cette perspective, nous chercherons à montrer dans quelle mesure la construction de cet ethos victimaire repose sur l'entrelacement de deux ordres complémentaires, celui de la raison et de la passion, pour faire advenir l'image que le locuteur se donne de lui-même en mélangeant donc logos et pathos; l'ethos faisant en effet osciller le discours « entre un pôle logico-cognitif et un pôle manipulatif-émotif » (Dascal 1999: 62). Ainsi, l'élaboration de cet ethos victimaire, c'est-à-dire l'image discursive d'une personne innocente symbolisant ici, dans une logique synecdochique particularisante ${ }^{10}$, la communauté musulmane elle-même, repose d'abord sur un discours d'objectivation du préjudice. Le dommage, pensé comme irrémédiable, fait en effet l'objet d'un discours pseudo-savant sous-couvert d'une argumentation étayée qui consiste à présenter le grief comme une réalité factuelle aisément démontrable. Ces apparences d'argumentaire rationnel sont d'ailleurs un trait spécifique de tout discours idéologique (Reboul, 1980) : on observe un masquage du jugement de valeur auquel on donne des apparences d'évidence scientifique irréfutable.

\subsection{Objectiver le préjudice : un discours étayé par les apparences d'un argumentaire rationnel}

\subsubsection{La construction d'une mémoire collective victimaire : quand les victimes écrivent leur Histoire}

L'histoire, comme marqueur du fait victimaire, joue un rôle d'envergure dans la production d'une mémoire collective, laquelle est constitutive de la vitrine numérique que donne à voir ces profils. Distincte de la mémoire historique ou "mémoire morte "11 (Halbwachs 1925), la mémoire collective se comprend au sens d'une lecture spécifique du passé qui favorise le façonnement identitaire d'une communauté. Ainsi, la "mémoire» du groupe étudié se présente comme le résultat de productions de discours qui sont le fait d'«entrepreneurs de mémoire», au sens d'individus " convaincus d'avoir une mission à accomplir et [qui] s'inspirent d'une éthique intransigeante en établissant une équivalence entre la mémoire qu'ils défendent et la vérité » (Pollak 1993 : 30).

Visant à présenter le peuple musulman, notamment à travers l'histoire coloniale, comme un objet de violence, les profils étudiés ont ainsi pour caractéristique commune de développer le récit d'une oumma ${ }^{12}$ assiégée et martyrisée. Présentée comme l'histoire «vraie » d'événements censurés par l'histoire institutionnelle française, la mémoire collective qu'ils produisent s'appuie sur un discours homogène qui investit les codes traditionnels de la mise en forme savante du récit historique (images 8 et 9). 


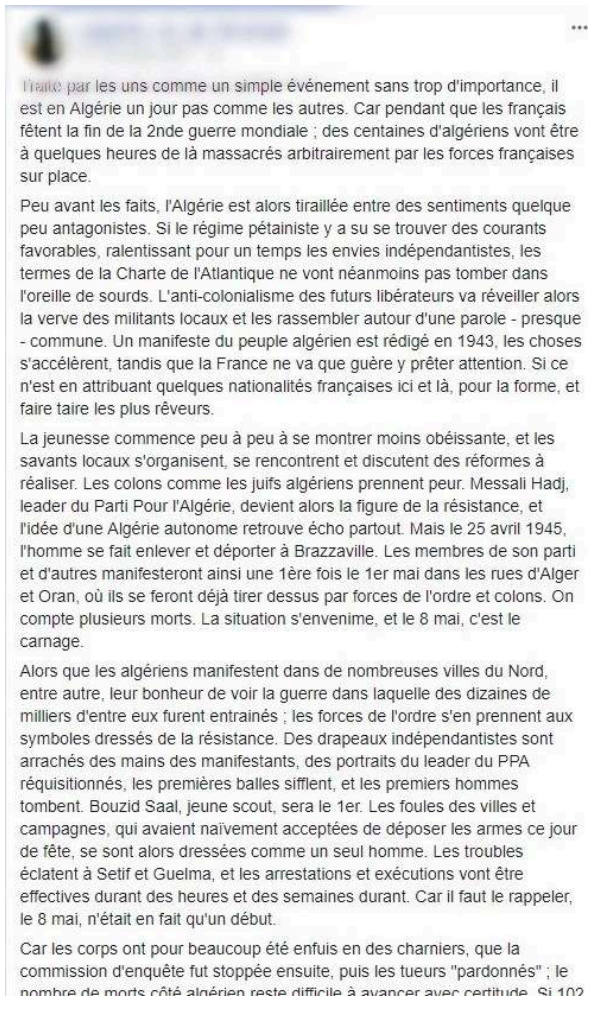

\section{Image 9}

Car les corps ont pour beaucoup été enfuis en des charniers, que la commission d'enquête fut stoppée ensuite, puis les tueurs "pardonnés" ; le nombre de morts côté algérien reste difficile à avancer avec certitude. Si 102 français furent tués en cette période, on parle de près de 30 à 45000 morts algériens en certaines sources. Si le chiffre est incertain, les moyens utilisés le sont beaucoup moins

A Kherrata, les colons retranchés en une forteresse vont tirer aveuglement sur la foule s'étant rassemblée et défilant dans les rues. L'armée, depuis des bateaux croiseurs et avions de guerre vont mitrailler et bombarder les populations avant d'y envoyer des hommes à terre. Sous prétexte de traquer les manifestants et autres rebelles et tueurs de français, miliciens, militaires et colons en arme vont tuer, torturer et humilier tout algérien trouvé en leur chemin. Femmes et nouveau-nés ne seront pas épargnés. Certaines seront violées sous les yeux de leurs familles quand elles ne sont pas prises nues en photo aux côté de colons souriant. Certains algériens sont jetés des falaises, quand les cadavres sont brulés à la chaux déposés par un balai incessant de camions bennes remplis de martyrs. Des hommes, dont certains sont encore aujourd'hui en vie et profitant d'une douce retraite vont, sous la bénédiction du général De Gaule commettrent des semaines durant les pires atrocités. Des villages vont être rasés, l'on tire à l'aide de fusils mitrailleurs à distance sur des populations désarmées.

La répression prenant fin officiellement le 22 mai. L'armée française va alors organiser des cérémonies de soumission où les algériens arrêtés vont se voir contraint de se prosterner devant le drapeau tricolore et répéter en chant qu'ils ne sont que des chiens. Certains, après ces cérémonies, seront même assassinés. L'historien algérien Boucif Mekhaled, raconte qu'à Kef El Boumba, il aurait vu "des français faire descendre d'un camion 5 personnes les mains ligotées, les mettre sur la route, les arroser d'essence avant de les brûler vivantes".

Alors qu'une guerre de 6 années s'arrêtait plus au Nord, une autre, qui allait conduire à l'indépendance du pays, avait bien commencé ce jour-là, le 8 mai 1945. Puisse le Très Haut accorder la meilleure des demeures dans l'Au delà aux croyants morts en les mains des pervers et injustes.

Renaud K.

22 Ce post est représentatif du discours «savant » produit ou relayé par et sur l'ensemble des comptes Facebook suivis. S'inscrivant bien dans la logique d'une mémoire collective 
victimaire, avec la guerre d'Algérie comme figure de proue de ce phénomène, ce texte dénonce la politique française de l'oubli et alimente la haine envers le colonisateur sous couvert d'une forme pseudo-historique. A cet égard, les dates ( 25 avril 1945 », « 8 mai $1945 »$ ), les noms de villes (Setif, Guelma, ou Alger) et les noms de victimes (Bouzid Saal) sont des procédés favorisant l'ancrage du récit dans le réel.

Par ailleurs, les données chiffrées, même approximatives et dont les sources ne sont pas citées, soutiennent une logique de décompte du nombre de victimes à déplorer : « 102 français » contre " 30 à 45000 algériens ». Ce bilan humain produit d'ailleurs un effet de saturation qui vise une lecture sous le prisme du registre compassionnel sur fond d'une rigueur scientifique censée garantir la véracité des faits. En outre, comme le relève Baudinière (2008), la question des exécutions sommaires occupe une place prépondérante dans ce type de littérature qui respecte deux règles du genre: les exécutions touchent des innocents ("un jeune scout») et les sévices subis sont particulièrement cruel ( 5 personnes les mains ligotées sont arrosées d'essence »). Enfin l'utilisation du témoignage, forme de discours censée véhiculer la subjectivité, est ici doublée d'une mobilisation de titres académiques (tel que "l'historien Boucif Mekhaled ») : ce n'est donc pas directement la victime qu'on fait témoigner mais le savant lui-même, de façon à donner du crédit au discours.

Souvent accompagné d'une photographie en noir et blanc (image 10), preuve visuelle de l'événement narré, ce type de post illustré contribue à nourrir l'ethos discursif de victime qui ne se réduit à pas sa dimension verbale dans la mesure où il fait aussi intervenir des "phénomènes sémiotiques extérieurs à la parole proprement dite " (Maingueneau 2013: 9); l'image étant bien sûr un élément de l'univers « technodiscursif» (Paveau 2015) qui influe sur la construction de l'ethos (image 9).

Image 10

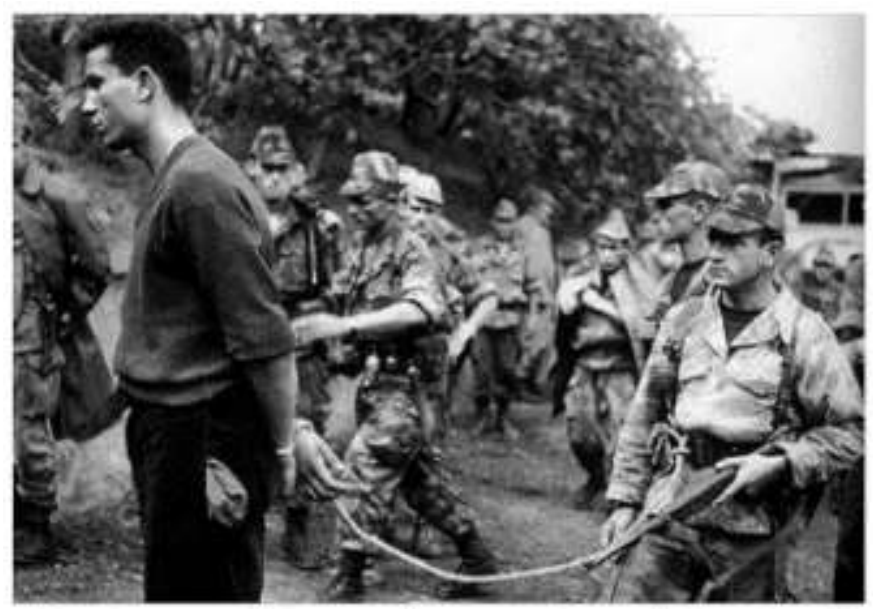

Comme montré par Paissa, «l'évènement historique devient le support d'une vision cristallisée du monde, d'un propos qu'on assène comme une évidence indiscutable, soustraite à la négociation, voire à la réfutation » (2016:11). Il est aussi le support d'un raisonnement qui vise à fournir une preuve par l'exemple: le recours à une argumentation par le récit historique, d'ailleurs érigé en preuve du fait victimaire, met ainsi en tension des données du passé visant à dresser les modèles explicatifs d'un présent inéluctable. Dans ce contexte, la mémoire collective joue bien un rôle essentiel dans la définition identitaire d'un groupe, par le biais d'une narration qui se veut 
objective, mais également dans la justification d'actions passées, présentes et futures du groupe ${ }^{13}$.

\subsubsection{Victimisation concurrentielle et argumentation par la comparaison : vers une palme de la souffrance}

Il n'y a pas de victime par essence: la victime n'a pas d'existence hors de la construction socio-discursive d'un préjudice. Or cette dernière a non seulement pour exigence l'affichage d'un dommage mais aussi la revendication d'une palme de la souffrance : il ne convient pas seulement de souffrir mais de souffrir plus que les autres et de s'attacher à le démontrer. Pour ce faire, le discours victimaire s'appuie sur une argumentation par la comparaison basée sur la dénonciation du non-respect de la règle de justice. Principe fondamental selon lequel les êtres d'une même catégorie essentielle doivent être traités de la même façon (Perelman 1990), cette règle est un élément caractéristique de la rhétorique victimaire avec le marqueur langagier du « deux poids deux mesures » (Koren, 2019), lequel souligne et dénonce une différence de traitement. On relève d'ailleurs comme dénominateur commun à l'ensemble des comptes étudiés, une disposition à se comparer avec ceux qui semblent jouir d'une meilleure situation en fournissant des parallèles saisissants (exemples 3 et 4 ).

(3) « Un ancien chef scout viole 50 fois 3 enfants, il écope seulement de 3 ans de prison. Normal les prisons sont peuplées de musulmans, après tout violer des gosses, c'est rien, c'est normal en France » (Post du 04/10/17).

Commentaire 1: "Quand tu fais la comparaison avec les peines de prison que prennent les musulmans juste pour apologie du terrorisme, y'a de quoi serrer wallah. »

Commentaire 2 : «A l'époque, j'ai pris cinq ans fermes pour un vol sans violence. Ah mais oui je suis musulman c'est vrai. »

(4) «Après l'interdiction du port du niquab, ils vont nous obliger à vendre l'alcool et le cochon ... si ça c'est pas de l'acharnement alors je sais pas ce que c'est mais une choses est sure, l'islam dérange dans ce pays de dépravé jusqu'à la moelle. Ni les juifs, ni les chrétiens ne seront jamais satisfaits de toi jusqu'à ce que tu suives leur religion » (Post du 14/12/17).

Commentaire 1 « Depuis quand on interdit aux juifs de pratiquer leur religion? Non c'est juste pour nous. »

Commentaire $2:$ «Patience mes frères et sœurs, nous en Syrie [...] nous sommes massacrés !!!»

Réponse $1:$ "J'ai honte de me plaindre quand je vois votre quotidien, c'est rien à côté de votre vie, qu'Allah vous préserve.»

La comparaison par le recours analogique à des données chiffrées (« un ancien chef scout viole 50 fois 3 enfants, il écope seulement de 3 ans de prison » versus «j'ai pris cinq ans fermes pour un vol sans violence») vise à dénoncer les différences de jugement des conduites en fonction de l'appartenance des auteurs. Interrogations rhétoriques (" depuis quand on interdit aux juifs de pratiquer leur religion?») et formules restrictives ("c'est juste pour nous ») s'entremêlent dans une logique de victimisation concurrentielle ou competitive victimhood (Vollhardt 2013), destinée à comparer les souffrances endurées par rapport à celles de l'exogroupe. De cette comparaison découle l'affirmation du caractère singulier de leur histoire victimaire, mettant en exergue une conscience de victime exclusive ou exclusive victim consciousness (ibid). En effet, la culpabilité de se comparer avec ceux qui pâtissent d'un plus triste sort ne se manifeste qu'au regard de la communauté musulmane elle-même (vivant dans un pays en guerre) : «J'ai honte de me plaindre quand je vois votre quotidien ». 


\subsubsection{L'argumentation d'autorité : la pratique citationnelle comme substitut de la} vérité

Sur les comptes Facebook étudiés, est visible la tentation de convoquer les grands esprits, ceux qui se sont illustrés dans divers domaines de la connaissance par le passé, afin d'exploiter ce prestige pour défendre l'idéologie djihadiste et son versant victimaire. Dans ce contexte, l'argument d'autorité est roi : consistant à se référer à une figure emblématique de savoir comme s'il s'agissait d'une preuve que cette opinion est vraie, la pratique citationnelle s'apparente à un refuge argumentatif qui offre une protection au locuteur à travers une forme d'enchaînement «logique». De cette manière, la référence à Platon (image 13), « personne n'est plus détesté que celui qui dit la vérité » sert ici de justification du sentiment victimaire sous couvert d'une instance d'autorité.

Image 11

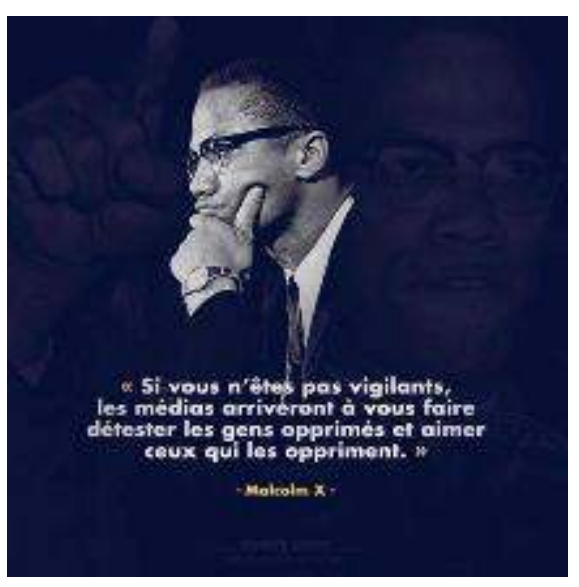

Image 12

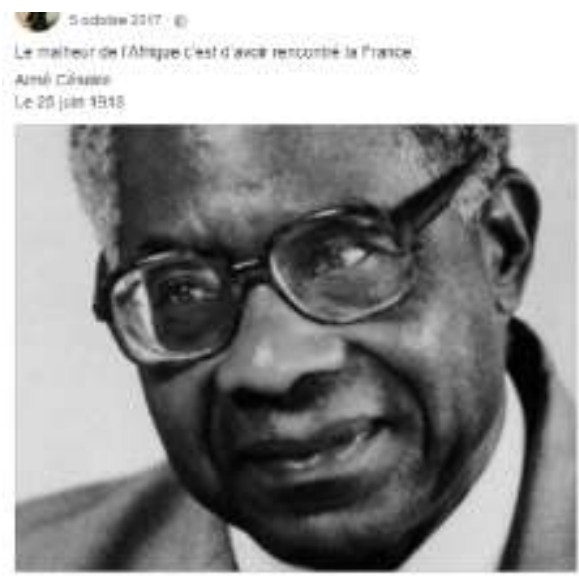

Haut du formulaire 


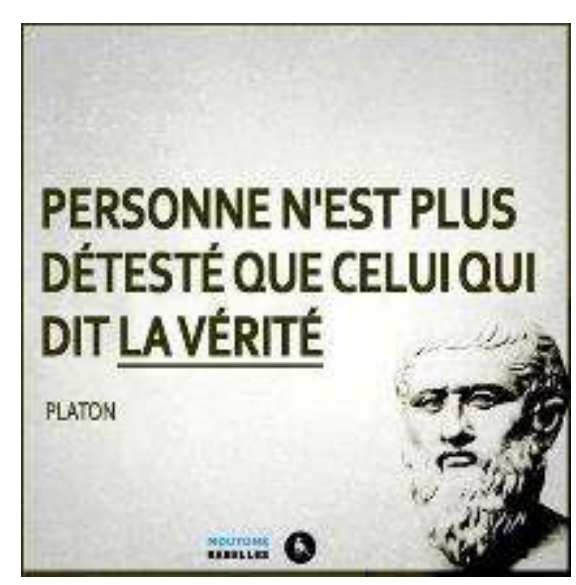

\subsection{Du logos au pathos : discours victimaire et intentionnalité pathémique}

\subsubsection{Discours pluri-sémiotique et rhétorique de la transparence}

Le discours de victimisation présente une dimension pathémique qui repose sur le recours à un discours pluri-sémiotique censé laisser transparaitre le monde tel qu'il est dans une exigence morale de transparence. Ainsi, sur fond d'argumentaire rationnel, le dispositif victimaire djihadiste glisse vers un dispositif argumentatif de type émotionnel, l'ethos de victime faisant osciller le discours entre pathos et logos qui se nourrissent mutuellement. Ici le logos est même mis au service du pathos : la rhétorique de la transparence, érigée en règle d'or et se manifestant par la divulgation de données sensibles, vise à communiquer la garantie d'une forme d'authenticité et d'accès à la connaissance : tout voir pour tout savoir. Au poids des mots s'ajoute le choc des images ou de vidéos sans filtres, leur contenu faisant l'objet d'un signalement sur Facebook pour contenu violent ou explicite (image 14). 


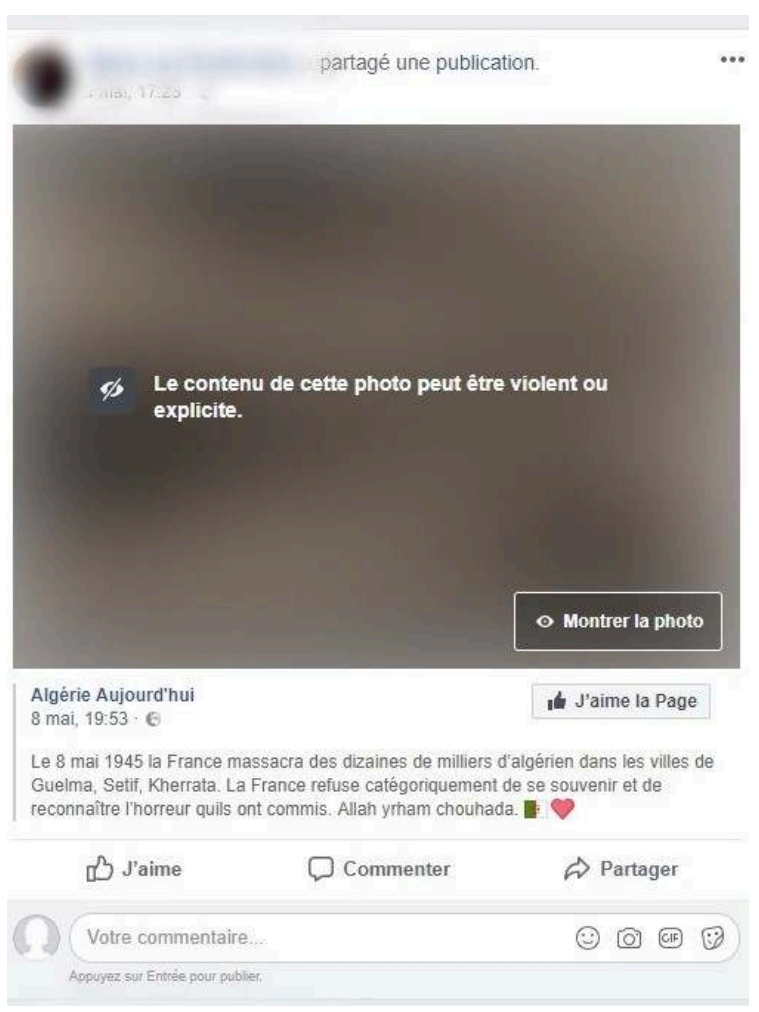

Précisons que la portée pathémique de ce discours repose sur deux registres, allant de l'émotion montrée à l'émotion suscitée. Dans le premier cas, le locuteur, pour communiquer l'émotion, se montre soi-même ému (5). Dans le second, il se contente d'exposer des faits censés susciter l'indignation (6).

(5) Il ne passe pas un jour sans massacres d'enfants. Mais rien de grave, «ce n'est qu'en Syrie ».

9 tués cette nuit à Khan el Sobol. Syria Charity, 24/05/16.

(6) Massacre en RDCongo dans le silence du reste du monde, on fait semblant de ne rien voir au moment que des millions des congolais sont massacrés...Les médias sont absents. Pfff. Nofi, 25/06/15.

\subsubsection{De l'indignation à l'engagement en ligne : les manifestations numériques de la solidarité victimaire}

31 L'étude de ce corpus nous invite à nous pencher sur les formes de "e-solidarité » djihadiste, les réseaux sociaux constituant des tremplins d'organisation de la solidarité. Appels aux dons en ligne, levées de fonds, actions et messages de soutien aux prisonniers ou aux malades font l'objet de publications destinées à s'entraider dans des situations d'urgence (images 15, 16 et 17). 
Image 15

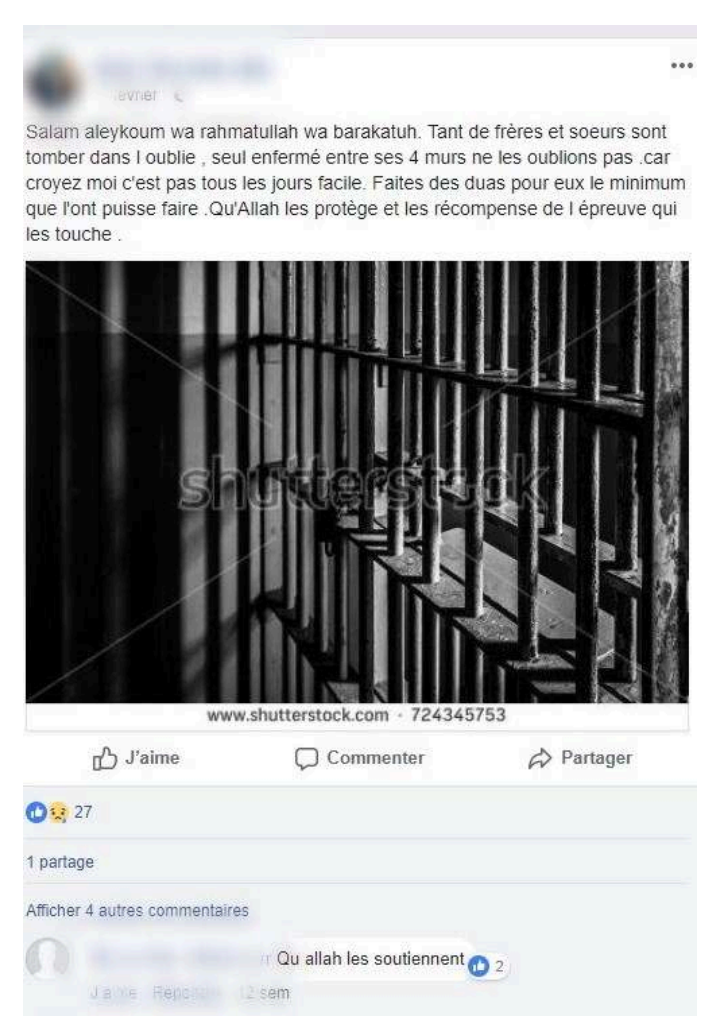

Image 16

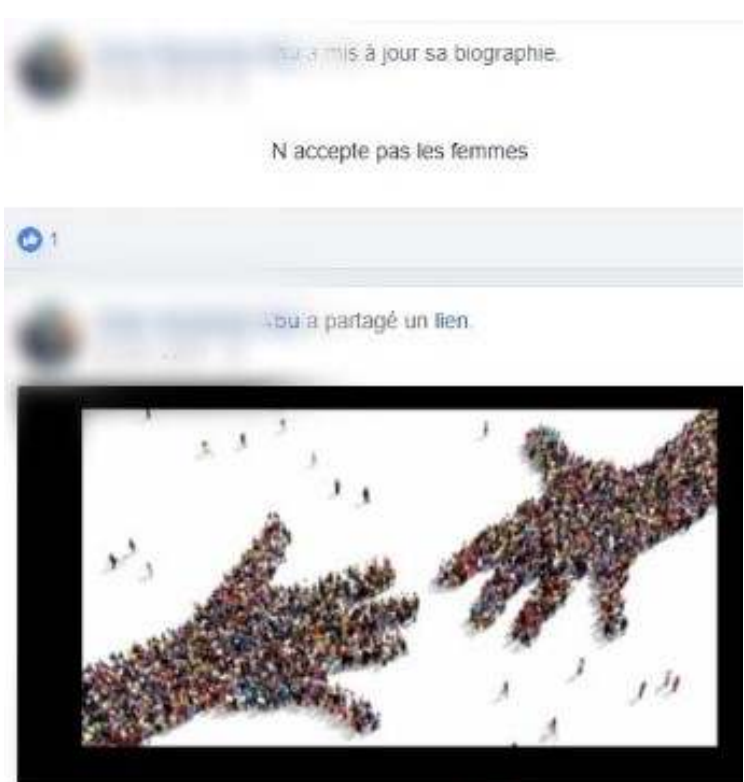

Aidons Oum Qassim - CotizUp.com $\odot$

Soutenez celte caunotle . particivez ou partagez au maximum ! COTIZUPCOM
d3 Jaime
$\square$ Commenter
$\Leftrightarrow$ Partager

a. 


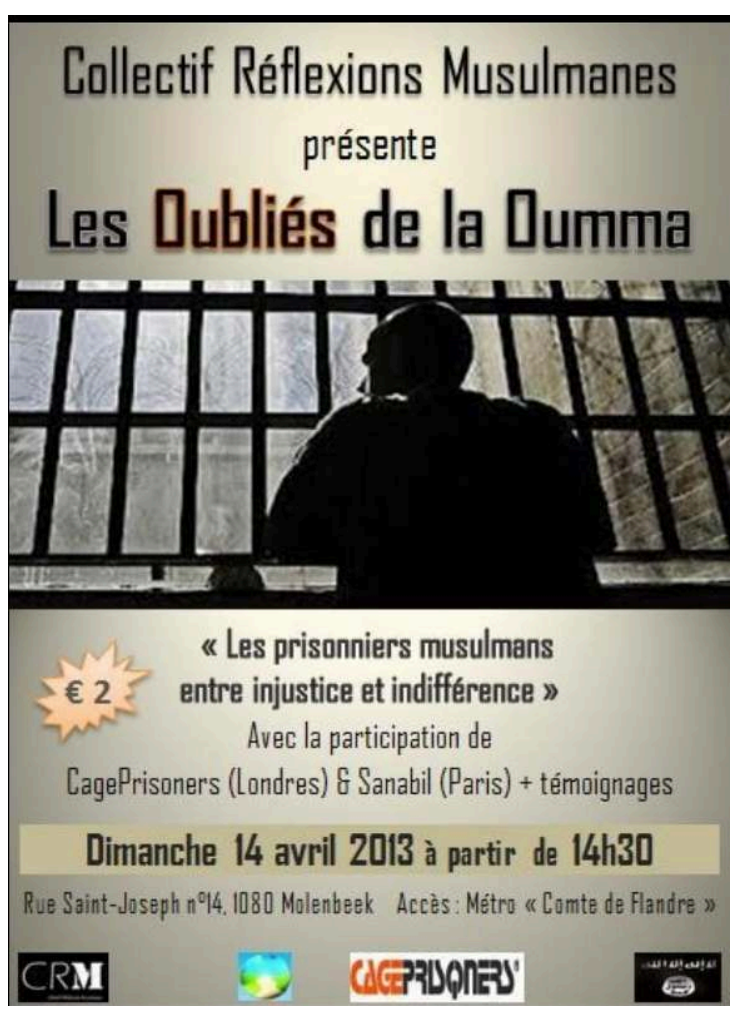

\subsection{La figure de victime comme facteur d'appartenance collective}

Le processus de construction d'une identité victimaire prend ici un tournant communautaire : la victime singulière s'attache en effet à former ou rejoindre une « communauté de destin» (Messu 2018 : 95), ici celle des vrais musulmans ou « antitaghout $~^{14}$ en opposition aux «mécréants » ou «kouffars». Dans ce contexte, le partage d'une expérience victimaire individuelle s'inscrit dans la revendication d'une identité collective: le «je» opprimé devenant plus largement l'étendard du musulman :

(7) En France, le musulman n'a pas le droit d'avoir froid. Je sors de chez moi, il fait froid, je cache légèrement mon visage avec mon jilbeb, les flics passent, forcément s'arrêtent devant moi, je leur explique que c'est pas un niquab, que j'ai froid juste, et je pars car je suis pressée, pas que ça à faire. Il me stoppe avec son camion de flics, la flic maquillée comme une fille de joie commence à me fouiller et me parle comme de la merde. J'avoue j'ai eu le malheur de dire "pays de facho ", elle me dit : "t'es pas contente, casses toi d'ici, personne te retient», si elle savait c'te bouffonne, bref je me suis mangée une amende pour rien donc les muslim surtout ne cachez pas votre visage même si vous claquez des dents, vous avez pas le droit d'avoir froid hein (Post du 12/10/17).

Commentaire 1 : Starfullah, que des pourritures, ils savent s'en prendre aux sœurs car ils flippent des frères !! Ecœurant pays de racistes !

Commentaire 21 : Oui ça devient de plus en plus dur de vivre ici. Ils profitent de leur pouvoir.

Réponse 1 : Oui ils ont fait exprès de me faire la misère, c'est tout, ils cherchent à me faire tomber. 
Réponse 2 : As salam alaykum restons patients, la victoire est plus proche qu'on ne croit pas, [...] ceci est une épreuve parmi d'autre... Courage à toi ainsi qu'à tous les opprimés.

Commentaire 3 : C'est connu qu'ils nous aiment pas, on pense pas comme eux et on vit pas comme eux.

Ce post, particulièrement représentatif de la logique de victimisation à l'œuvre dans ce corpus numérique, est le vecteur d'une forme d'«identité affirmative » ou « fierté » qui trouve son origine dans les années 1980 sur la base d'une dénonciation des discriminations subies (Messu 2018: 155). Ici, le partage social d'une expérience émotionnelle individuelle («je cache mon visage avec mon jilbeb, les flics [...] forcément s'arrêtent devant moi ») soutient la réclamation d'une identité de victime partagée par une communauté ( «les muslim surtout ne cachez pas votre visage même si vous claquez des dents »). Le dispositif argumentatif déployé ici repose sur un raisonnement inductif explicite puisque du récit particulier découle une loi générale tandis que le sentiment de persécution oscille entre ces deux pôles: si l'acharnement est présenté comme ciblé (« ils cherchent à me faire tomber »), il est le symptôme d'un mal plus général (« en France, le musulman n'a pas le droit d'avoir froid »). Au-delà de l'expression d'une plainte, cette publication nourrit la revendication d'appartenance à un groupe qui « prétend se distinguer de l'ensemble sociétal, de la masse des autres, sur la base d'un puissant critère : celui de [son] état victimaire » (Messu 2018 : 155). Elle est ainsi plus largement la manifestation numérique de la figure du «surmusulman », qui fait entrer en collusion l'humiliation de l'islam par l'Occident avec une blessure plus subjective (Benslama 2016).

On retrouve la vision dichotomique du monde promue par Daesh et basée sur une série d'oppositions (le bien/ le mal, "ils»/ «nous»), cette logique mettant en valeur la communauté d'appartenance, tout en dévalorisant l'autre, ici source de rejet: "c'est connu qu'ils nous aiment pas, on pense pas comme eux et on vit pas comme eux. » La différence avec cet autre dominant et le sentiment de persécution lié à cette différence nourrit la cohésion du groupe tout comme cette dernière facilite en parallèle le processus individuel de construction victimaire : «En se pensant appartenir à une communauté, un ensemble, un groupe victimisé, l'individu va pouvoir intégrer personnellement l'attribut. Cela devient pour lui une caractéristique identitaire. [...] La catégorisation substantielle a opéré » (Messu $2018: 155)$.

Ces comptes radicalisés constituent ainsi des "communautés interprétatives " (Fish 1980), au sens de groupes qui partagent et affichent les mêmes codes et valeurs. C'est dans ce cadre que le recours fréquent à l'ironie se développe sous la forme d'un blâme par la louange (image 18).

Argumentation et Analyse du Discours, 23 | 2019 


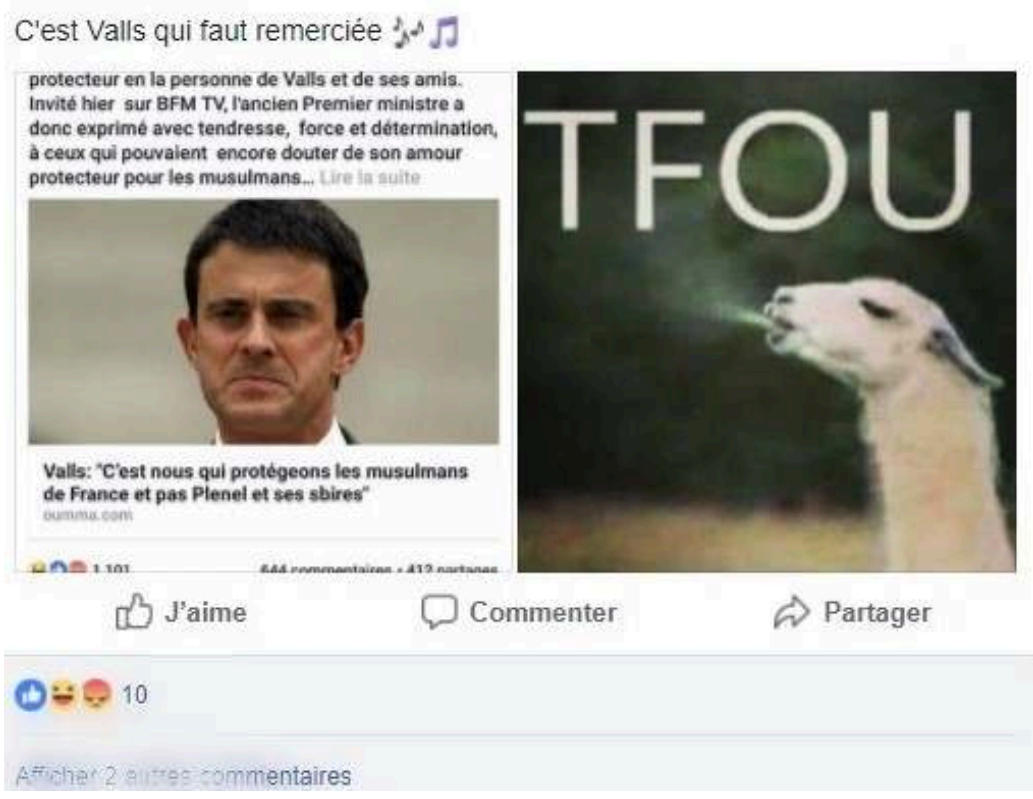

Symptomatique du procès ironique, dont le propre est de n'être décelable qu'au sein d'une communauté capable de réévaluer une assertion en la devinant incompatible avec ce qu'il sait de la vision de l'auteur, ce post fonctionne sur le mode du clin d'œil. Révélateur du phénomène de connivence ironique, lui-même fondé sur un processus d'inclusion/ exclusion, la publication fait l'apologie implicite du terrorisme sous le couvert du registre humoristique. En effet, l'expression «c'est Valls qu'il faut remercier" suivie d'une note de musique fait allusion au refrain de l'anasheed djihadiste Ma vengeance ${ }^{15}$, appelant à commettre des attentats en France. Moyen privilégié d'accès à l'idéologie d'un groupe, l'ironie permet bien d'opposer «pensée dominante (généralement sa cible) et pensée minoritaire $»^{16}$.

\section{Justifier l'action violente : de l'ethos victimaire à l' ethos du justicier}

\subsection{L'impossible performativité du statut victimaire comme ressort du désir de vengeance}

La figure de victime, on l'a vu, s'inscrit par essence dans une quête de reconnaissance statutaire destinée à lui faire bénéficier d'un traitement spécial. Or ici, l'échec de ce processus, c'est-à-dire l'impossibilité d'obtenir une réparation symbolique, semble être le levier d'un glissement identitaire lorsque la victime présumée affiche ses prétentions au statut de justicier. En effet, du sentiment d'être victime d'une injustice qui n'est en outre pas reconnue comme telle, découle une propension au désir de vengeance. Dans le cas de la radicalisation, cette logique débouche sur une collusion entre les notions de victime et de bourreau qui se mue en coulissement stratégique de l'une à l'autre : ainsi 
« la dialectique du bourreau et de la victime [...] s'efface au profit de l'occultation, du voilement et finalement de la disparition de la victime, comme le voile du magicien vient recouvrir le lapin, objet appelé à s'évanouir » (Messu 2018 : 31).

Alors que la logique vengeresse est aujourd'hui rejetée du champ des émotions avouables, elle fait pourtant l'objet d'une publicisation ${ }^{17}$ dans la djihadosphère. En effet, on fait ici étalage d'un ressentiment qui s'exprime sous la forme d'un discours justicier et sur lequel repose le discours de la vengeance ${ }^{18}$; la déficience de la justice justifie le recours à la violence en tant qu'outil compensatoire au service d'une forme d'autojustice. Nombres de publications légitiment ainsi la contre-attaque au motif d'un rétablissement d'équilibre sur la base de la Loi du Talion (image 19 et exemple 8).

Image 19

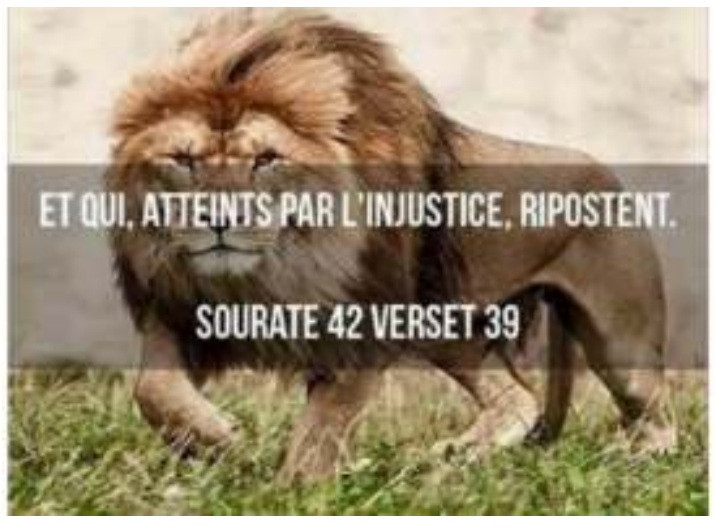

(8) Moi je suis pas un rat, provoque moi et tu verras qui t'étranglera (Post du 23/04/18)

Ces deux exemples illustrent la tentative sémio-discursive (en alliant le texte à l'image dans le premier cas) de rapprocher la vengeance de la notion de justice, l'idée étant que la riposte infligée au coupable est défensive et équivalente au dommage subi par la victime présumée. Le discours de la vengeance n'hésite pas ici à faire référence à une valeur transcendante en guise de justification de l'action violente, la mention d'une sourate (image 19) ayant le rôle d'un argument d'autorité : les terroristes ne sont en ce sens rien d'autre que les bras vengeurs d'une religion qu'ils estiment bafouée. Perçue comme un acte de dévouement à la communauté musulmane, la vengeance est explicitement encouragée à travers la diffusion de l'anasheed, Ma Vengeance, véritable leitmotiv de ce chant de propagande: "Leur guerre impitoyable/ Est la seule responsable/ Notre vengeance est louable/ Nous sommes inarrêtables [3 fois] ». Le martèlement de cet adjectif "inarrêtable " nous semble intéressant en tant que marqueur d'une mutation d'ethos en cours, comme si le passage à l'action conférait un pouvoir d'émancipation à la victime. Dès lors, l'expression assumée de la vengeance semble s'inscrire dans le prolongement logique du discours victimaire djihadiste tout en en parachevant les contours. Porte d'entrée vers un nouvel ethos, celui du justicier, ce dernier est métaphorisé sur les comptes djihadistes par le lion (image 20), symbole des soldats du califat et signe de ralliement pour ses partisans 2.0. 
Image 20

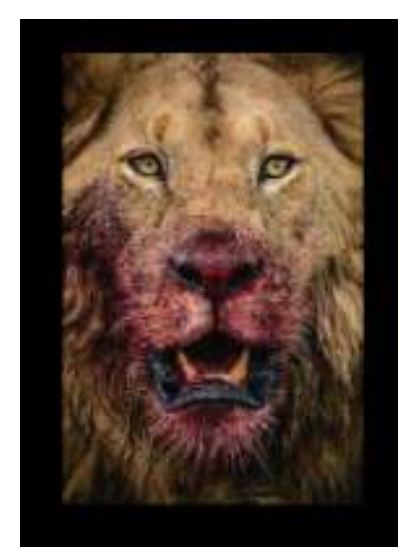

\subsection{La fabrique discursive de l'ennemi : un double processus de "dévictimisation »}

\subsubsection{Renverser le stigmate}

Notre recherche nous permet à ce stade de postuler d'un double glissement identitaire à l'œuvre sur ces profils tant en termes de présentation de soi (ce que je dis de moi), avec le passage de la victime au justicier, que de désignation (la manière dont je suis dit par l'autre) notamment pour les profils les plus avancés dans le processus de radicalisation. Ce glissement identitaire s'appuie sur un travail de renversement du stigmate, lequel concrétiserait la formation d'une identité collective (celle des djihadistes) aux antipodes de l'image qu'ils présupposent être véhiculée d'eux (celle de fous).

Nous concevons donc ce déplacement identitaire en opposition à l'identité du groupe premier qui s'opère via une conversion des représentations stigmatisantes initialement attribuées. En cela, nous insistons à la suite de Plumauzille et Rossigneux-Méheust (2014) sur la capacité tactique des agents à se saisir de leur stigmate pour les transformer, une prise de conscience pouvant en effet "amener les stigmatisés euxmêmes à renverser leur stigmate [...], soit en se distanciant de l'identité première, soit en contribuant à la formation d'une identité nouvelle notamment au niveau des normes et des valeurs » (ibid. : 18).

Pour ce faire, on relève un processus de différenciation ou d'identisation, par lequel la personne radicalisée marque sa différence en niant être ce qu'elle a l'air d'être à la manière des conspirationnistes sur la base de l'adage «les apparences sont trompeuses" (image 21). A cet égard, on relève la répétition d'une formule de réfutation «je ne suis pas fou » (exemple 9), suivie d'une accusation "c'est toi qui es laxiste (image 22) comme "manœuvre de rétorsion» (Amossy, Koren, Saltykov 2018). Compris au sens de « retourner un argument contre celui qui s'en est servi» (Dupriez 1980 : 400), ce mécanisme discursif vise à combattre l'ennemi en lui «arrachant ses propres armes» (Angenot 1982: 219). Stratégie de réparation par excellence, l'argument du tu quoque est ainsi mobilisé pour retourner le blâme à l'expéditeur, c'està-dire délégitimer les détracteurs. 


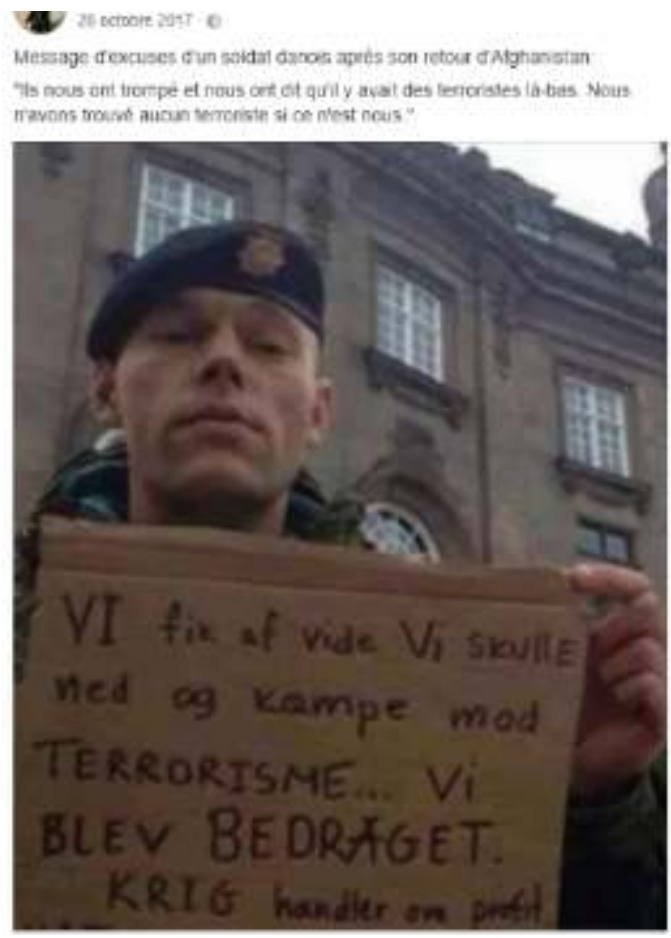

(9) «Je ne suis pas un fou, pourtant j'ai fait plus d'un an chez les fous, d'Allah je suis fou, pour lui je suis prêt à tout, ne me trahis pas ou tu finiras sous mes roues » $(11 / 04 / 19)$

Image 22

En se présentant donc comme les victimes d'idées reçues, les profils radicalisés entreprennent de convertir le stigmate, ce qui leur permet de se défendre dans une optique d'exonération de leurs responsabilités mais aussi de réparation d'image.

\subsubsection{Diaboliser la victime occidentale : du bouc-émissaire sans visage au " criminel qui s'entête »}

Puisque le partage d'anasheeds revient fréquemment sur les comptes Facebook étudiés, il nous a semblé pertinent de nous pencher sur ce type de corpus pour appréhender le traitement discursif de la victime occidentale dans la propagande djihadiste auxquels sont soumis les profils suivis. Or, on note une véritable évolution de la production d' anasheeds francophones entre l'avant et l'après 14 juilllet 2016. Jusqu'à cette date, les anasheeds associaient la figure de l'ennemi à celle d'une victime coupable. Parmi le spectre des nominations la désignant, on relève dans Avance avance ${ }^{19}$, "traitres", «criminels qui s'entêtent", «abrutis", «ennemis à exterminer» ou à «faire exploser ». L'autre est le terreau d'un déferlement d'images d'une violence extrême visant à le déshumaniser et encourager la guerre à son encontre.

Or, si les anasheeds produits après cette date demeurent des concentrés d'exaltation à tuer le mécréant occidental, celui-ci relève davantage d'un bouc émissaire, payant le prix fort d'une politique menée par les dirigeants occidentaux. Le tristement célèbre chant $M a$ Vengeance diffuse l'image d'une foule anonyme et d'une cible nécessaire, à travers lesquelles on s'attaque indirectement au ministre de l'intérieur, Manuel Valls: 
Ma kalash est armée/ Les civils désarmés/J'élimine des Français. Le discours de justification des victimes semble donc avoir évolué sous la pression d'une opinion publique particulièrement choquée par les images diffusées dans les médias après l'attentat de Nice.

Pour autant, la construction par la communauté djihadiste de son persécuteur repose dans les deux cas sur un processus de " dévictimisation » qui consiste à déshumaniser la victime, facilitant ainsi l'établissement d'une distance psychologique nécessaire pour déclencher la violence contre un groupe.

\subsection{Du bourreau au héros : le martyr ou la promesse d'une alternative à la figure de victime}

Le martyre, promu par la propagande djihadiste, recouvre selon cette idéologie la concrétisation la plus évidente d'une vertu héroïque : l'argument par le sacrifice ${ }^{20}$ suppose ici le renoncement à sa propre vie au nom d'un combat sacré contre l'injustice. On relève à cet égard une fascination à prédominance masculine pour les terroristes tombés en fomentant un attentat. Cette admiration se manifeste sur les réseaux sociaux par de véritables monuments aux morts numériques (images 23 à 27) : certains comptes Facebook abondent en photos ou en reportages biographiques, leur permettant une meilleure connaissance de leurs héros.

Image 23

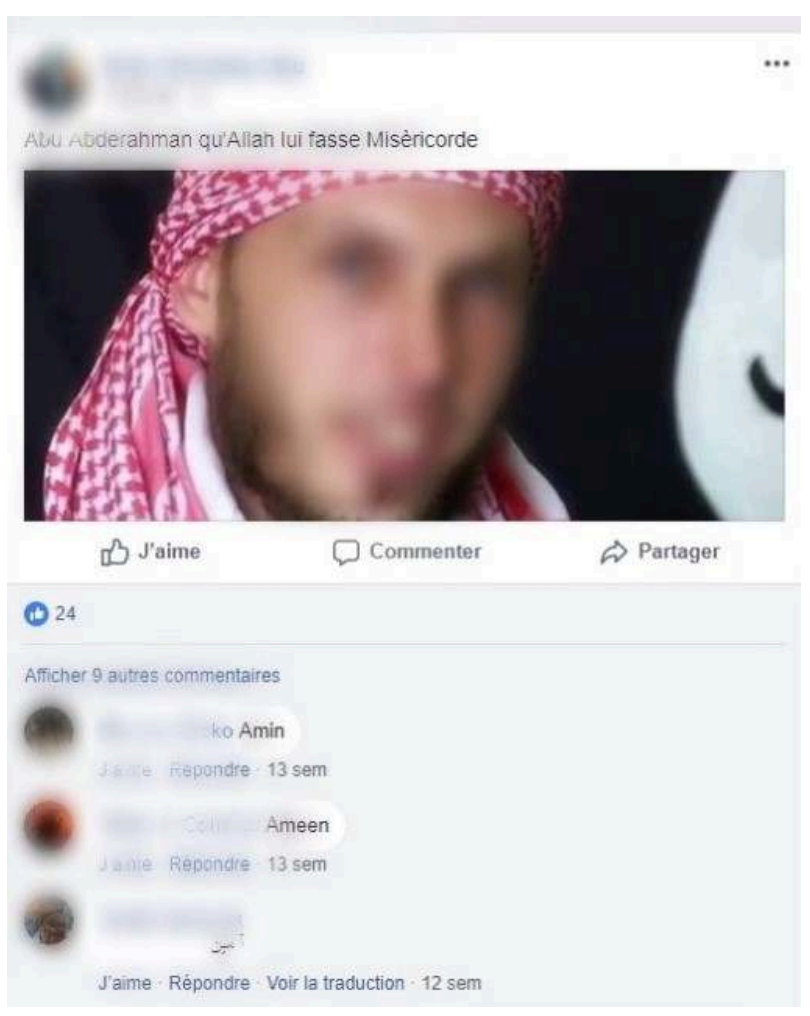


Image $24^{21}$

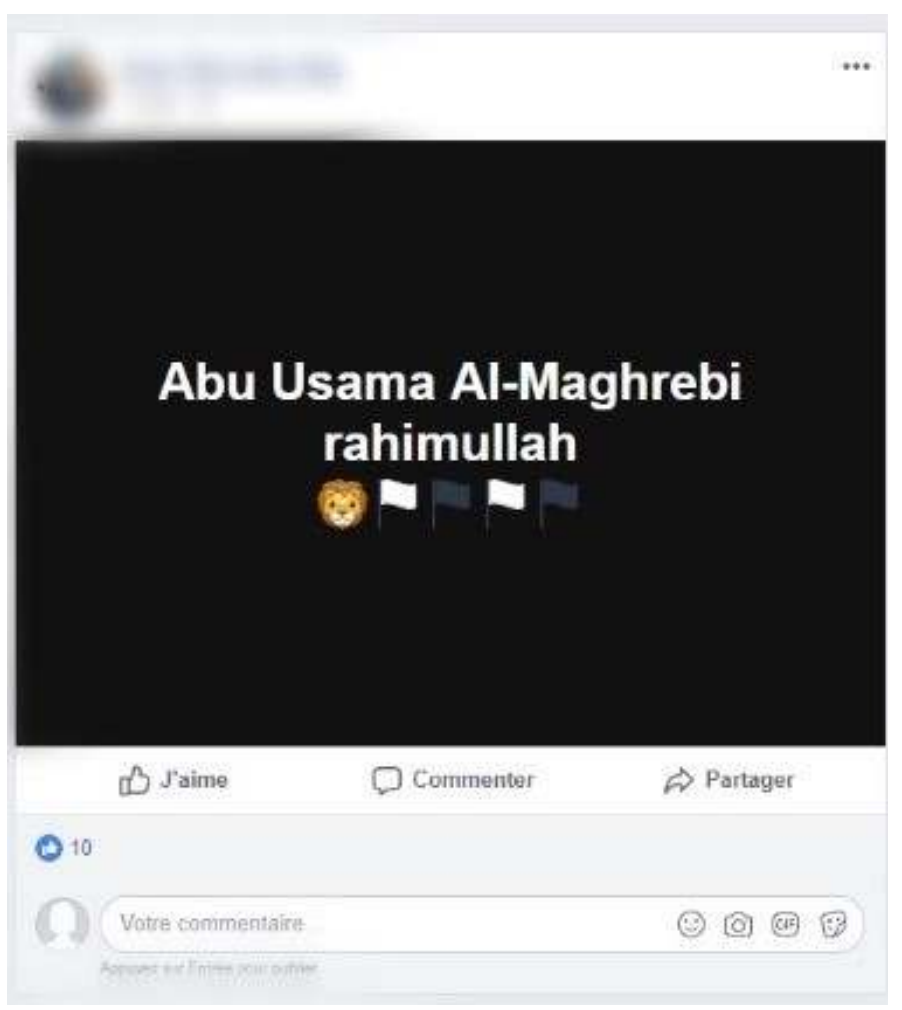

Image 25

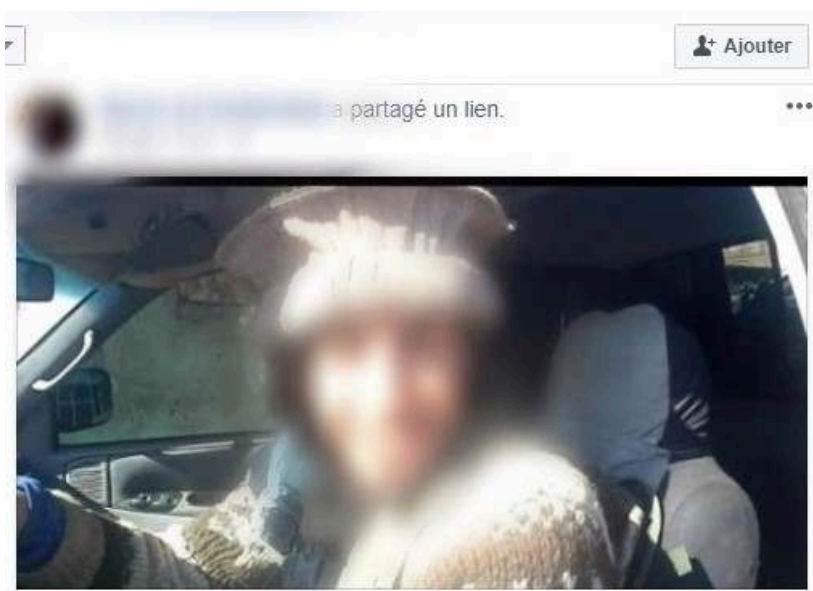

Imagen: Attentats de Paris: Abdelhamid Abaaoud, cerveau présumé des ...

Se encontró en Google desde youtube.com

IMAGES.GOOGLE.COM
ㄱ. J'aime
๑ Commenter
$\Rightarrow$ Partager

(1) Votre commentaire...
Appuyez sur Entée pour publer.




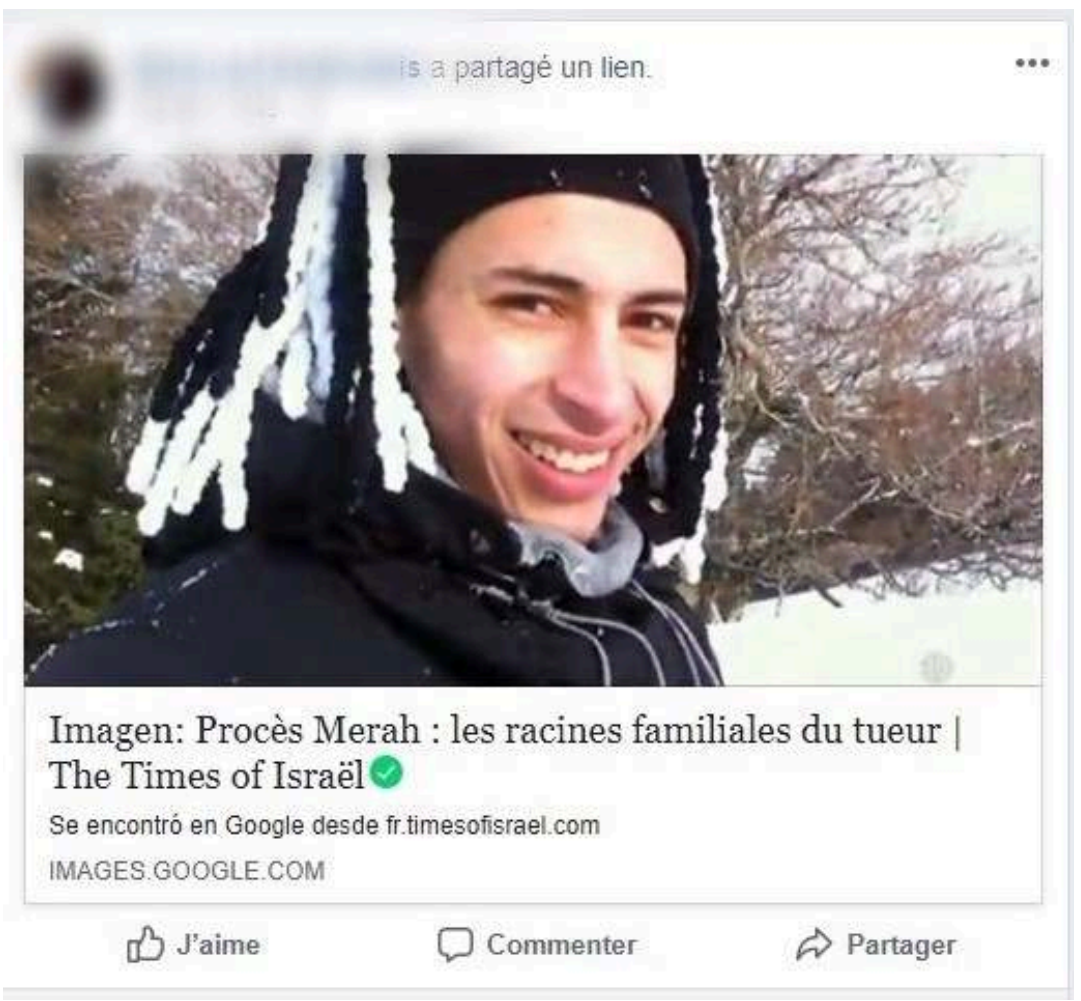

Images 27

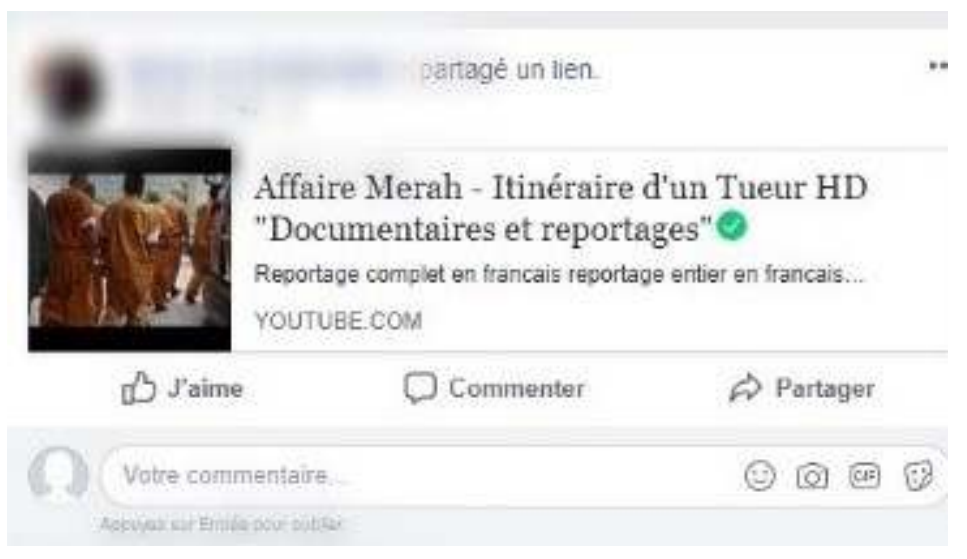

(10) « La mort! Pour nos martyrs » (24/04/18)

(11) « Faut saigner les porcs, c'est pour les frères en or, moi il n'y a que Dieu que j'adore et je ne crains pas la mort » $(26 / 04 / 18)$.

L'exemple 10 cristallise l'un des ressorts principaux de ce culte des martyrs, à savoir sa dimension fédératrice ("pour nos martyrs »). Considéré comme un héros qui dépasse son intérêt au profit du bien commun, il devient, par la nature de son geste, exemplaire : les martyrs représentent donc plus que de simples modèles, «ils font de l'imitation un devoir à leur égard et deviennent ainsi les destinataires et les inspirateurs concrets des attitudes religieuses ou éthiques » (Albert 1999). Ce devoir est verbalisé par l'emploi de l'impératif : «faut saigner les porcs, c'est pour les frères en or » (exemple 11), visant à s'inscrire dans leur lignée. La valeur qui justifie et stimule l'acte sacrificiel n'est autre qu'une forme de transcendance puisque la mort présente ici 
un motif religieux (ibid.). Précisons que les bénéficiaires supposés de cet (auto)sacrifice sont d'abord la communauté des vrais-musulmans dans une optique réparatrice de leurs souffrances, l'humanité tout entière dans une visée purificatrice, et surtout Allah dont les djihadistes considèrent être les serviteurs. Comme montré par Koren, les mobiles altruistes réhabilitent ici le sacrificateur et valorisent son choix de résister à l'oppression pour le bien de ses semblables en inversant « les connotations mortifères dévalorisantes de l'acte criminel » (2018:6). Or, comme souligné par (Messu $2018: 14)$ :

Lorsque le héros et la victime se rejoignent ou plus exactement, lorsque les images, les représentations mentales significatives de l'un et de l'autre, se fondent en un syntagme nominal [...] nous assistons à l'émergence sociale d'une nouvelle catégorie d'appréhension et de compréhension du monde social lui-même. [...] En effet, le rapprochement des deux vocables [...] va autoriser une forme d'héroïsation de la victime et faire advenir à la positivité la figure plutôt négative de la victime.

La victime se confond donc ici avec le héros qui revendique le rétablissement de la justice par son action, à travers les épreuves qu'il affronte. Le processus d'héroïsation de la victime s'apparente dès lors à une échappatoire au statut de victime présentée par Daesh comme une figure passive qui subit ses malheurs sans pouvoir y faire face. A l'inverse, le héros s'apparenterait à un sujet doté de vouloir et de pouvoir.

\section{Conclusion}

A travers cette étude du discours victimaire djihadiste, nous avons montré que les ressorts mis en œuvre témoignent d'une logique explicative, ou manière exclusive de voir le monde, de la part des scripteurs. C'est pourquoi, au-delà d'une réponse rationnelle et argumentée, c'est aussi la perception du monde, selon la lecture victimiste, qui est à déconstruire. S'agissant d'une forme d'idéologie, il s'agirait dès lors de bouleverser cette grille de lecture ou déchiffrage du monde (victime/ coupable, nous/ les autres), peut-être davantage que les idées liées à cette grille de lecture ${ }^{22}$. Par ailleurs, nous souhaitons attirer l'attention sur un paradoxe à prendre en compte dans la production de contre-discours ou dans la réflexion à mener pour prévenir la radicalisation : en effet si la victimisation n'est pas loin de concerner chaque prise de parole dans la djihadosphère, notons pourtant un mépris affiché de ses membres pour la figure de victime ; «il fait sa victime » ou "c'est une grosse victime » étant des insultes courantes à l'encontre des ennemis de la communauté. Dès lors, il convient d'interroger les éléments de langage officiels de certains contre-discours et les représentations parfois ambivalentes qu'ils véhiculent du jeune djihadiste, entre acteur de la radicalisation mais aussi victime d'un discours d'embrigadement ${ }^{23}$. 


\section{BIBLIOGRAPHIE}

Aïm, Olivier et Pauline Escande-Gauquié. 2016. « L'écranalyse ou la mise en réflexivité des écrans à l'ère de leur multiplication ", Interfaces numériques 5, URL : https://www.unilim.fr/interfacesnumeriques/2953

Albert, Jean-Pierre. 1999. La fabrique des héros (Paris : Éditions de la Maison des Sciences de l'Homme)

Amossy, Ruth, Roselyne Koren \& Maria Saltykov. 2018. « Une approche argumentative de la réparation d'image : Jean-Luc Mélenchon aux présidentielles de 2017 », Langage et société 164-2, 117-135

Angenot, Marc. 1982. La parole pamphlétaire. Typologie des discours moderne (Paris : Payot)

Ascone, Laura. 2018. La radicalisation à travers l'expression des émotions sur internet, Thèse soutenue le $22 / 11 / 18$

Benslama, Fethi. 2016. Un furieux désir de sacrifice. Le surmusulman (Paris : Seuil)

Crick, Nicki \& Jennifer Grotpeter. 1995. "Relational Aggression, Gender, and Social-Psychological Adjustment", Child Development 66-3, 710-722

Dascal, Marcelo. 1999. «L'ethos dans l'argumentation : une approche pragma-rhétorique », Amossy, Ruth (dir.). Images de soi dans le discours. La Construction de l'ethos (Lausanne : Delachaux et Niestlé), 61-73

Denis, Benoit. 2007. « Ironie et idéologie », COnTEXTES 2, URL : http://journals.openedition.org/ contextes/180; DOI : 10.4000/contextes. 180 .

Dupriez, Bernard. 1980. Gradus. Les procédés littéraires (Dictionnaire) (Paris : 10/18)

Fish, Stanley. 1980. "Is There A Text in This Class?" (Harvard U. P), 147-174

Garric, Nathalie \& Julien Longhi. 2012. «Atteindre l'interdiscours par la circulation des discours et du sens ", Langage et société 144, 65-83

Hawker, David \& Michael Boulton. 2000. “Twenty Years' Research on Peer Victimization and Psychosocial Maladjustment: A Meta-Analytic Review of Cross-Sectional Studies”, Journal of Child Psychology and Psychiatry 41, 441-455

Halbwachs, Maurice. 1925. Les Cadres sociaux de la mémoire (Paris : Albin Michel)

Khosrokhavar, Farhad. 2014. Radicalisation (Paris : Éditions de la Maison des Sciences de l'Homme) Koren, Roselyne. 2018. « De quelques éloges littéraires ou idéologiques de la violence mortifère : quand la fin justifie les moyens ", Exercices de rhétorique 11, URL : http:// journals.openedition.org/rhetorique/671

Koren, Roselyne. 2019. Rhétorique et Éthique : du jugement de valeur (Paris : Classiques Garnier)

Lahrache, Fouad. 2016. « Être plus musulman que musulman : Réflexions à partir du livre Un furieux désir de sacrifice, le surmusulman », VST - Vie sociale et traitements 132-4, 28-34

Longhi, Julien. 2018. Du discours comme champ au corpus comme terrain (Paris : L'Harmattan)

Maingueneau, Dominique. 2013. «L'ethos : un articulateur », COnTEXTES 13, DOI : 10.4000/ contextes. 5772 
Maingueneau, Dominique. 1999. «Ethos, scénographie, incorporation », Amossy, Ruth (éd.). Images de soi dans le discours. La construction de l'ethos (Lausanne : Delachaux \& Niestlé), $75-100$

Messu, Michel. 2018. L'ère de la victimisation (Paris : Editions de l'Aube)

Naït-Bouda, Faïza. 2017. « Renversement du stigmate et agir créatif : identité para-journalistique des journalistes pigistes », Revue française des Sciences de l'Information et de la Communication 11

Paissa, Paola 2016. « Exemple historique et argumentation : autour de la reconnaissance du massacre du 17 octobre $1961 »$, Argumentation et Analyse du Discours 16, URL : http:// journals.openedition.org/aad/2160

Paveau, Marie-Anne. 2012. «Facebook, aphorisme et narcissisme », La pensée du discours, URL : https://penseedudiscours.hypotheses.org/235

Paveau, Marie-Anne. 2013. «L'intégrité des corpus natifs en ligne. Une écologie postdualiste pour la théorie du discours », Les cahiers de praxématique 59, 65-90

Perelman, Chaïm \& Lucie Olbrechts-Tyteca. 1983. Traité de l'argumentation. La nouvelle rhétorique, $4^{\mathrm{e}}$ édition, (Bruxelles : Éditions de l'Université de Bruxelles)

Perelman, Chaïm. 1990. Ethique et droit (Bruxelles : Editions de l'Université de Bruxelles)

Plumauzille, Clyde \& Mathilde Rossigneux-Méheust. 2014. « Le stigmate ou la différence comme catégorie utile d'analyse historique », Hypothèses 17-1, 215-228

Pollak, Michael. 1993. Une identité blessée. Études de sociologie et d'histoire (Paris : Métailié)

Reboul, Olivier. 1980.Langage et idéologie(Paris : PUF)

Renaut L, Ascone L, 2019 (sous presse). « Contre-discours au discours de haine djihadiste. De l'expression de la conflictualité à la fabrique du doute », Semen 47

Vollhardt, Johanna Ray. 2013. « 'Crime against Humanity’ or ‘Crime against Jews'?

Acknowledgment in Construals of the Holocaust and Its Importance for Intergroup Relations », Journal of Social Issues 9-1, 144-161

\section{NOTES}

1. Il est à noter que la victimisation a aussi son importance sur le chemin du désengagement via les récits d'ex-djihadistes " repentis » dont le discours repose lui aussi sur une logique ambiguë de victimisation, entre rejet et recours implicite. (Renaut et Ascone 2019)

2. La seule mention équivalente au terme de victime est celui d' " opprimé ».

3. On ne peut évoquer l'Afghanistan sans prendre en compte l'invasion soviétique en 1979 pendant la Guerre froide durant laquelle les Etats-Unis, en guise de riposte, financent alors les moudjahidines : soutenus notamment par le Pakistan et l'Arabie Saoudite, ceux-ci contribuent à faire de l'Afghanistan le terreau du salafisme djihadiste en transformant le pays en un gigantesque camp d'entraînement pour les apprentis terroristes. Quant à la question irakienne, précisons que ce pays est gouverné jusqu'en 2003 par le sunnite Saddam Hussein, bien connu pour opprimer la communauté chiite. Suite à la révolution iranienne en 1980, l'Irak, dont la situation financière est particulièrement mise à mal par son opposition à l'Iran, initie en 1990 un embargo du Koweït avorté par la coalition américaine. En outre, après la chute du régime de Saddam Hussein en 2003 avec l'invasion américaine, les chiites, soutenus par les Etats-Unis s'emparent du pouvoir, et répriment à leur tour la communauté sunnite en procédant à de 
nombreuses arrestations de personnes liées ou non au régime de Saddam Hussein. Ce renversement de la situation nourrit alors le ressentiment des sunnites contre les chiites et c'est dans cette conjecture tendue que le déclin de l'armée irakienne en 2014 conduit à la naissance de groupes jihadistes sunnites, dont Daesh. Enfin, en 2011, se développe en Syrie, une révolte de la jeunesse du pays contre la corruption et les entraves aux droits humains. S'ensuit une révolte civile et la formation d'une opposition militaire au régime du musulman alaouite Bashar alAssad, notamment avec la Free Syrian Army, alors violemment réprimée. Nombre de djihadistes étrangers rejoignent la Syrie pour participer à la rébellion contre le régime d'Assad qui mène alors quatre guerres de front: une contre ses ennemis internes, plus ou moins démocratiques, une contre Daesh, une contre les États-Unis et leurs alliés ; et une aux côtés de ses alliés chiites (Liban et Iran) contre les puissances sunnites comme l'Arabie Saoudite.

4. Depuis 2014, ce département Communication et Médias créé par Daesh diffuse des revues: Dabiq publiée en anglais (15 numéros), Dar al-Islam rédigée en français (dix numéros) et depuis septembre 2016 Rumiyah déclinée en huit langues (13 numéros). On retrouve aussi des vidéos et enregistrements de chants à destination des musulmans non-arabophones afin de promouvoir l'idéologie djihadiste.

5. Les nasheeds ou nashîds sont des chants religieux musulmans, polyphoniques et sans accompagnement.

6. Notre recherche étant centrée sur un phénomène discursif (victimisation) et non sur la caractérisation du fonctionnement du discours numérique, nous espérons nous libérer du piège des corpus clos, élaborés à partir d'une hypothèse générique, et accéder à une saisie plus globale et précise des phénomènes socio-historiques (Garric et Longhi 2012).

7. Dans le contexte de la radicalisation djihadiste, un mécréant désigne une personne qui n'adhère pas à la religion considérée comme la seule vraie, ici l'Islam. On relève comme équivalent la dénomination kuffar ou infidèle.

8. Le terme hèbs en arable signifie prison.

9. Image accompagnée du texte suivant: «En \#Birmanie, on martyrise à mort des musulmans \#rohingya pour leur religion. Si les médias ne veulent pas en parler, c'est à vous de le faire ! Les réseaux sociaux sont AUSSI faits pour cela... »

10. On nomme la partie pour signifier le tout, contrairement à la synecdoque généralisante, où l'on nomme le tout pour signifier la partie.

11. Pour Halbwachs, la mémoire historique renvoie à la mémoire d'événements que l'individu n'a pas vécus lui-même et qui n'auraient aucun impact direct sur l'identité d'un groupe.

12. L'oumma ou oummat ou ummat (arabe : أمّة), qui signifie « communauté » ou « nation », est la communauté des musulmans, indépendamment de leur nationalité.

13. Voir à ce sujet les travaux de Bartlett (1932) montrant que l'histoire victimaire d'un groupe est continuellement remaniée par ses membres afin de satisfaire au mieux ses intérêts actuels.

14. Dans le vocabulaire islamique, le Taghout ou Tâghût représente tout ce qui est adoré en dehors d'Allah. Quant à la façon de se présenter comme " anti-Tâghût ", elle est corrélée à la croyance de la nullité de l'adoration d'un autre qu'Allah, de juger mécréant celui qui la pratique, et même de considérer comme ennemi celui qui s'en rend coupable.

15. Ma Vengeance est le titre d'un clip de propagande produit par al-Hayat Media Center, dans lequel l'organisation dévoile des images d'exécutions et d'attentats : http://www.non-stopzapping.com/actu/divers/brahim-abdeslam-vise-francois-hollande-dans-un-clip-de-

propagande-de-daesh-video-34466

16. On retrouve « un trait commun entre l'analyse idéologique [...] et la posture ironique, en ce que l'une et l'autre sont particulièrement efficaces pour s'en prendre aux manifestations discursives de la domination » (Denis $2007: 8$ ).

17. Nous entendons le phénomène de publicisation dans son sens littéral correspondant à l'action de publier ou rendre public, ce qui est le cas des posts Facebook publics étudiés. 
18. Précisons qu'il y a dans l'origine étymologique même des termes «vengeance » et " justice » un lien étroit, vengeance venant du latin vindicare (réclamer en justice) et « justice » de judicare (dire le droit).

19. Le nasheed Avance, avance, véritable hymne de guerre contre la France " croisée ", est diffusé sur internet dans le communiqué audio de revendication des attentats du Bataclan puis dans la vidéo Paris s'est effondrée.

20. Voir les travaux de Koren (2018) sur la transformation du discours victimaire en justification de l'action violente via le recours à l'argument par le sacrifice : « La primauté donnée à l'axiome la fin justifie les moyens est à l'origine du recours à l'argument par le sacrifice. [...] Perelman et Olbrechts-Tyteca classent ce type d'argument, contre toute attente, dans la catégorie quasilogique des raisonnements comparatifs, espace argumentatif où la raison pratique se rapproche considérablement de la raison théorique. Le sacrifice serait le prix que l'actant serait prêt à payer pour avoir droit aux gratifications de la fin » (Koren $2018: 4$ ).

21. Mort en 2014, Abdulaziz al-Mahdali, connu sous le nom d'Abou Usamah al-Maghrebi, était l'un des principaux commandants militaires de l'EI en Iraq et au Levant.

22. Si les stratégies de disqualification du discours victimaire et plus largement du discours de haine djihadiste, n'ont pu être suffisamment explorées dans ce cadre de cet article, elles font l'objet d'une publication sous presse (Renaut et Ascone 2019).

23. C'est d'ailleurs le propos du site gouvernemental On te Manipule mais aussi du clip Ils te disent.

\section{RÉSUMÉS}

Cette recherche se propose d'interroger, d'un point de vue discursif, la construction du dispositif victimaire djihadiste et d'analyser comment ce dernier est mis au service d'une justification de l'action violente, à partir d'un corpus numérique de profils caractérisés comme radicalisés. La première partie de l'article explicite la démarche adoptée, l'adossement théorique ainsi que méthodologique, et propose une caractérisation thématique du corpus à partir de la notion de grief. Dans une seconde partie, nous analysons les ressorts sémio-discursifs sur lesquels repose la construction d'une identité victimaire propre au phénomène de radicalisation djihadiste. Enfin, nous cherchons à montrer dans quelle mesure le discours de victimisation djihadiste vise à promouvoir la vengeance par la construction d'un ethos de justicier. A cet égard, nous interrogeons l'impossible performativité du statut victimaire comme ressort du ressentiment et étudions le processus de "dévictimisation » visant autant à déshumaniser les victimes du terrorisme que les terroristes eux-mêmes.

This research aims to question the construction of the jihadist victim system, and to analyze how it is used to justify acts of violence, based on a digital corpus of radicalized social media profiles. The first section of the article explains the approach, the theoretical and methodological background, and proposes a thematic characterization of the corpus based on the notion of grievance. We then analyze the semio-discursive elements on which the construction of a victim identity specific to the phenomenon of jihadist radicalization draws. In the final section, we show to what extent the discourse of jihadist victimization aims to promote revenge through the construction of a figure of avenger. In this regard, we will examine the impossible performativity of the status of victim springing from resentment and study the process of "devictimisation" aimed at dehumanizing both the victims of terrorism, and the terrorists themselves. 
INDEX

Keywords : ethos, jihadist radicalization, martyrdom, victim discourse

Mots-clés : discours victimaire, ethos, martyr, radicalisation djihadiste

\section{AUTEUR}

\section{LAURÈNE RENAUT}

Université de Cergy-Pontoise (UCP) 